\title{
Cachazo-He-Yuan theory for several fields
}

\author{
C. S. Lam๑* \\ Department of Physics, McGill University, Montreal, Quebec, Canada H3A 2T8 \\ and Department of Physics and Astronomy, University of British Columbia, \\ Vancouver, British Columbia, Canada V6T $1 Z 1$
}

(Received 20 April 2020; accepted 7 July 2020; published 22 July 2020)

\begin{abstract}
The Cachazo-He-Yuan formula was originally proposed to describe on-shell scattering of particles from a single massless field. We present a method to modify it to include several interacting scalar fields, all possessing different masses and possibly off-shell momenta. The method is applied to Yukawa interactions between a number of scalar nucleons and pions, and to the $\phi_{1} \phi_{2} \phi_{3}$ coupling of three different scalar fields. Composite models constructed from existing theories can be used to broaden the scope of the method. The modification is applied to describe Compton scattering from a massive particle and to photon bremsstrahlung. It is also employed to generalize the disk function $Z$ and the sphere function $J$.
\end{abstract}

DOI: $10.1103 /$ PhysRevD.102.025018

\section{INTRODUCTION}

Inspired by string theory in the zero Regge slope limit, Cachazo-He-Yuan (CHY) came up with a formula for the tree amplitude of several massless field theories, valid in any number of spacetime dimension [1-3]. Like the string theory, the CHY formula is given by a multiple integral over the complex plane, possessing Möbius invariance. A set of scattering equations plays an important role in this formalism. Propagators are now closely related to the scattering functions in the scattering equations, and vertices are tied up with the global structure of the amplitude.

As originally conceived, the $\mathrm{CHY}$ formula describes the scattering of a massless scalar field with $\phi^{3}$ coupling, the pure Yang-Mills theory, and Einstein's gravity. These are all massless fields with massless external momenta. Because the formula is applicable in any number of spacetime dimensions, dimensional reduction can be used to construct many other theories in lower dimensions $[4,5]$.

The correctness of the CHY formula was proved in [6] and directly verified by computations [7-19]. Properties and solutions of the scattering equations have been investigated [20-34], soft and collinear limits have been derived [35-39], and loop computations have been attempted [40-47]. It has been reformulated as a string theory $[48,49]$, and as a double-cover integral [50]. The relations between different $\mathrm{CHY}$ theories have been studied using

\footnotetext{
*Lam@physics.mcgill.ca
}

Published by the American Physical Society under the terms of the Creative Commons Attribution 4.0 International license. Further distribution of this work must maintain attribution to the author(s) and the published article's title, journal citation, and DOI. Funded by SCOAP. differential operators and scattering forms [51-59]. The connection between $\mathrm{CHY}$ and string theories has also been investigated [60-64].

As interesting and as novel as this new formulation is, it would not be helpful to high energy physics unless it can describe the Standard Model. That would require a number of additional developments not contained in the original formula. The formulation must be broadened to include off-shell amplitudes in order to coincide with field theory, and for loops to be computed. Nonzero masses must be included because most particles in nature are massive. The usual way to do that relies on the validity of the $\mathrm{CHY}$ formula in any number of dimension, so four-dimensional masses and off-shell momenta can be obtained from momenta in extra dimensions [65-67]. This works in some special cases, but not in general, because one cannot be assured that all correct propagators can be obtained this way. For example, the propagators of a massive $\phi^{3}$ theory cannot be so obtained if the total number of external particles far exceeds the total number of extra dimensions. This is further explained in the Appendix A.

There is, however, a different method to generate a massive $\phi^{3}$ theory and/or off-shell momenta that guarantees correct propagators in any number of dimensions, achieved simply by modifying the scattering equations $[68,69]$. The method has also been used to extend the CHY Yang-Mills theory off-shell [70].

To reproduce the Standard Model, massive fermions in the fundamental representation must be included. That proves to be quite difficult in the CHY formalism although some interesting progress has been made [71,72].

Nature contains many massive particles in a variety of interactions, so the $\mathrm{CHY}$ formula must also be generalized to describe them, on-shell and off-shell. In this article we 
propose a method to do so for scalar particles, again by modifying the scattering equations. We shall work out in detail the Yukawa coupling of several scalar nucleons and pions, all with different masses. In the special case of an infinite pion mass, this degenerates into the $\phi^{4}$ theory. We also discuss how to couple three different scalar fields via $\phi_{1} \phi_{2} \phi_{3}$ interaction. In addition, a very useful method to construct composite models from existing theories will be discussed. In this way we can for example construct a model in which two nucleons of different masses couple to form a dinucleon resonance, and the resonances can interact with one another via an exchange of pions. Beyond scalar particles we also take a first look at photons. Compton scattering and bremsstrahlung from a charged massive scalar particle can both be computed this way.

A disk function $Z$ and a sphere function $J$ have been introduced to connect massless field theory amplitudes to string amplitudes $[60,73,74]$. The modification of scattering functions mentioned above naturally leads to a possible modification of the $Z$ and $J$ functions. It would be interesting to see whether such a modification can be used to generate new string amplitudes and/or new effective field theories.

In Sec. II, the general setup of the method is described, together with illustrations taken from the two known cases: massless on-shell $\phi^{3}$ scattering [3], and massive on-shell or off-shell scattering [68]. This method is then applied to the Yukawa coupling of one massive scalar nucleon and one massive pion in Sec. III, and to the Yukawa coupling of several massive scalar nucleons and pions in Sec. IV. Three field coupling $\phi_{1} \phi_{2} \phi_{3}$ is taken up in Sec. V, and the construction of composite models is discussed in Sec. VI. Compton scattering and bremsstrahlung from charged scalar particles will be considered in Sec. VII and Appendix C. Modified $Z$ and $J$ functions will be discussed in Sec. VIII, and a short summary is included in Sec. IX. Appendix A explains why extra dimensions cannot be simply used to obtain massive and/or off-shell amplitudes in four dimensions, and Appendix B proves a general covariant condition needed for the $\mathrm{CHY}$ formula to be extended.

\section{THE CHY $\phi^{3}$ THEORY AND ITS GENERALIZATION}

\section{A. Equation of motion}

Consider a meromorphic function $F(\sigma)$ defined by

$$
\begin{aligned}
F(\sigma) & =\sum_{i \neq j=1}^{n} \frac{a_{i j}}{\left(\sigma-\sigma_{i}\right)\left(\sigma-\sigma_{j}\right)}=\sum_{i=1}^{n} \frac{1}{\sigma-\sigma_{i}} \sum_{j \neq i, j=1}^{n} \frac{a_{i j}}{\sigma-\sigma_{j}} \\
& :=\sum_{i=1}^{n} \frac{\hat{f}_{i}(\sigma)}{\sigma-\sigma_{i}}
\end{aligned}
$$

where $\sigma_{i}, a_{i j}=a_{j i}$ are arbitrary complex parameters subject to the constraints $a_{i i}=0$, and

$$
\sum_{j=1}^{n} a_{i j}=0(1 \leq i \leq n) .
$$

Under a Möbius transformation,

$\sigma \rightarrow \frac{\alpha \sigma+\beta}{\gamma \sigma+\delta}, \quad \sigma_{i} \rightarrow \frac{\alpha \sigma_{i}+\beta}{\gamma \sigma_{i}+\delta}, \quad \alpha \delta-\beta \gamma=1, \quad 1 \leq i \leq n$,

and as a consequence of the constraint, $\hat{f}_{i}(\sigma)$ can be shown to transform covariantly as

$$
\hat{f}_{i}(\sigma) \rightarrow \lambda^{2} \hat{f}_{i}(\sigma), \quad \text { where } \lambda=(\gamma \sigma+\delta) .
$$

The residue of $F(\sigma)$ at $\sigma=\sigma_{i}$ is

$$
f_{i}=\sum_{j \neq i, j=1}^{n} \frac{a_{i j}}{\sigma_{i}-\sigma_{j}}=\hat{f}_{i}\left(\sigma_{i}\right) .
$$

Given an $a_{i j}, F(\sigma)=0$ for all $\sigma$ if and only all its residues are zero, which is so if and only if the $n$ parameters $\sigma_{i}$ are solutions of the $n$ scattering equations $f_{i}=0$. In light of covariance, three $\sigma_{i}$ can be chosen arbitrarily, so only $n-3$ of these $f_{i}$ can be linearly independent. This linear dependence is encoded in the three sum rules:

$$
\begin{aligned}
\sum_{i=1}^{n} f_{i} & =0, \\
\sum_{i=1}^{n} f_{i} \sigma_{i} & =0, \\
\sum_{i=1}^{n} f_{i} \sigma_{i}^{2} & =0 .
\end{aligned}
$$

Both Eq. (4) and Eq. (6) are known to be true for the original CHY massless on-shell $\phi^{3}$ theory [1-3]. They are also true for the massive off-shell $\phi^{3}$ theory [68]. That they are generally true for any $a_{i j}$ satisfying Eq. (2) is shown in Appendix B. It is this covariant property that allows the $\mathrm{CHY}$ formula Eq. (7) to be extended to cover many other field theories.

We shall refer to $F(\sigma)=0$ as the equation of motion (EOM) because it generates all the scattering equations, and because it resembles the Klein-Gordon equation in ordinary field theory. In a field theory, the EOM is a differential equation true for all spacetime points $x$. In the CHY theory, it is an algebraic equation true for all complex variables $\sigma$.

\section{B. CHY amplitudes}

Solutions of the scattering equations determine the $n$-particle tree amplitude $A_{n}$. One way to express that is through the $\mathrm{CHY}$ formula 


$$
A_{n}=\left(-\frac{1}{2 \pi i}\right)^{n-3} \oint_{\Gamma} \sigma_{(r s t)}^{2}\left(\prod_{i=1, i \neq r, s, t}^{n} \frac{d \sigma_{i}}{f_{i}}\right) \mathcal{I}_{n},
$$

where $\Gamma$ is a clockwise contour encircling all $f_{i}=0$, with $f_{i}$ given by Eq. (5), and $\mathcal{I}_{n}$ is chosen so that $A_{n}$ is invariant under the Möbius transformation Eq. (3). Möbius invariance permits a choice of three arbitrary gauge constants $\sigma_{r}$, $\sigma_{s}, \sigma_{t}$ in the integrand that does not affect the outcome of $A_{n}$ in Eq. (7).

Each $f_{i}$ factor plays the role of an inverse scalar propagator, and the $\mathcal{I}_{n}$ factor plays the role of interaction vertices. For scalar theories which we shall concentrate mostly on in this article,

$$
\mathcal{I}_{n}=\frac{1}{\sigma_{(12 \cdots n)} \sigma_{(\alpha)}},
$$

where $\alpha=\left(\alpha_{1} \alpha_{2} \cdots \alpha_{n}\right)$ is a permutation of $(12 \cdots n)$ determined by the specific Feynman diagram under consideration. The quantity $\sigma_{\left(p_{1} p_{2} \cdots p_{k}\right)}$ stands for the product $\prod_{i=1}^{k} \sigma_{p_{i} p_{i+1}}$, with $k+1 \equiv 1$, and $\sigma_{a b}=\sigma_{a}-\sigma_{b}$. When spin-1 particles are involved, $\mathcal{I}_{n}$ would be different. We shall discuss a special case in Sec. VII.

It is implicitly assumed in Eq. (8) that every Feynman tree diagram is written as a planar diagram, with the external lines ordered clockwise and cyclically in the natural order $1,2, \ldots, n$. The amplitude Eq. (7) is that of a single Feynman diagram, or that of a sum of several or all Feynman diagrams, all depending on what $\sigma_{(\alpha)}$ is. See Sec. II E for more discussions on that point.

The parameters $a_{i j}$ are so far arbitrary except for the constraint in Eq. (2). They will be chosen to reproduce the correct propagators in a given theory. When the external lines are ordered in the way described in the last paragraph, every propagator takes on the form $1 /\left(k_{S}^{2}-m_{S}^{2}\right)$, where $k_{S}=\sum_{i \in S} k_{i}$ is the total external momentum of a set $S$ of $\leq(n-2)$ consecutive external lines, $k_{i}$ is the outgoing momentum of line $i$, and $m_{S}^{2}$ is the (square) mass of the propagator. In a CHY scalar theory given by Eq. (7), the inverse propagators are related to the parameters $a_{i j}$ by the formula [68]

$$
k_{S}^{2}-m_{S}^{2}=\sum_{i, j \in S} a_{i j}
$$

Different theory specifies different $m_{S}^{2}$, and it is from this propagator equation that all $a_{i j}$ are determined. Once $a_{i j}$ are determined this way, we must still check that the symmetry condition $a_{i j}=a_{j i}$ and the covariant condition Eq. (2) are satisfied. Otherwise Eq. (7) cannot be used. Moreover, we must make sure that Eq. (9) is self-consistent in the following sense.

For any set $S$ of consecutive external lines, let $\bar{S}$ be its complementary set, consisting of all the external lines not in $S$. Since $S$ and $\bar{S}$ share the same propagator in a tree diagram, we must make sure that $k_{S}^{2}-m_{S}^{2}=k_{\bar{S}}^{2}-m_{\bar{S}}^{2}$. With momentum conservation, $k_{S}=-k_{\bar{S}}$, so this is satisfied if and only if

$$
m_{S}^{2}=m_{\bar{S}}^{2}
$$

for every set $S$. We shall refer to that as the complementary condition.

The two cases in which $a_{i j}$ are known are the original CHY massless on-shell $\phi^{3}$ theory [3] and the massive off-shell $\phi^{3}$ theory [68]. They will be reviewed in the following subsections. After that, we shall elaborate on how the propagator condition Eq. (9) can be used to determine $a_{i j}$ for other theories, and then proceed to apply the method to a scalar Yukawa theory whose "scalar nucleon" and "pion" possess different masses. In the limit of an infinite pion mass, this becomes the $\phi^{4}$ theory. This Yukawa theory can be generalized to include many kinds of nucleons with different masses, provided they interact with one another only through the exchange of pions. In these theories, $a_{i j}$ determined by the propagator condition can be made to obey the symmetry and the covariant conditions, as well as Eq. (10). We will also discuss a theory with three fields interacting according to $\phi_{1} \phi_{2} \phi_{3}$. The propagator condition once again determines its $a_{i j}$ for arbitrary masses of the three fields, but it turns out that such a $a_{i j}$ satisfies the required conditions only when the three masses are identical. Composite models and photons, as well as modified disk and sphere functions, will be discussed in later sections.

\section{C. $\phi^{3}$ theory, on-shell and massless}

This is the original CHY theory [3]. With $k_{i}^{2}=0$ and $m_{S}=0$, Eq. (10) is automatically satisfied. The choice of $a_{i j}$ for $i \neq j$ is

$$
a_{i j}=k_{i} \cdot k_{j}, \quad i \neq j .
$$

The symmetry condition $a_{i j}=a_{j i}$ is obviously true. Owing to momentum conservation and the massless requirement, the covariant requirement Eq. (2) is also satisfied because $\sum_{j=1}^{n} a_{i j}=-k_{i}^{2}=0$. The propagator condition Eq. (9) with $m_{S}^{2}=0$ also follows because

$$
\sum_{i, j \in S} a_{i j}=\sum_{i \in S} \sum_{j \in S} k_{i} \cdot k_{j}=k_{S} \cdot k_{S}=k_{S}^{2}
$$

\section{D. $\phi^{\mathbf{3}}$ theory, off-shell and with mass $\mathrm{m}$}

This situation was studied in [68]. The solution with $a_{i i}=0$ is

$$
a_{i j}=k_{i} \cdot k_{j}+\rho_{i j}-\frac{1}{2} \mu_{i j}, \quad \text { for } i \neq j .
$$

The $\rho_{i j}=\rho_{j i}$ term is responsible for off-shell extensions, and the $\mu_{i j}=\mu_{j i}$ term is needed for a nonzero mass $m$. Specifically, for $n>4$, 


$$
\begin{aligned}
\rho_{i, i \pm 1} & =\frac{1}{2}\left(k_{i}^{2}+k_{i \pm 1}^{2}\right), \quad \mu_{i, i \pm 1}=m^{2}, \\
\rho_{i, i \pm 2} & =-\frac{1}{2} k_{i \pm 1}^{2}, \quad \mu_{i, i \pm 2}=-m^{2}, \\
\rho_{i j} & =0, \quad \mu_{i j}=0, \quad \text { for }|j-i|>2 .
\end{aligned}
$$

The indices $i \pm 1$ and $i \pm 2$ could turn out to be nonpositive, or larger than $n$. In that case they should be interpreted to have a value $\bmod n$.

For $n \leq 4, j$ could be equal to or less than two lines away on both sides of $i$. In that case Eq. (13) should be used to add up both sides to get the correct result for $\rho_{i j}$ and $\mu_{i j}$.

Since all the propagators have mass $\mathrm{m}^{2}$, Eq. (10) is satisfied. With this $a_{i j}$, the covariant condition Eq. (2) is satisfied because

$$
\begin{aligned}
\sum_{j=1}^{n} a_{i j}= & \sum_{j \neq i, j=1}^{n}\left(k_{i} \cdot k_{j}+\rho_{i j}-\frac{1}{2} \mu_{i j}\right) \\
= & -k_{i}^{2}+\rho_{i, i+1}+\rho_{i, i-1}+\rho_{i, i+2}+\rho_{i, i-2} \\
& -\frac{1}{2}\left(\mu_{i, i+1}+\mu_{i, i-1}+\mu_{i, i+2}+\mu_{i, i-2}\right) \\
= & -k_{i}^{2}+\frac{1}{2}\left(k_{i}^{2}+k_{i+1}^{2}-m^{2}\right)+\frac{1}{2}\left(k_{i}^{2}+k_{i-1}^{2}-m^{2}\right) \\
& -\frac{1}{2}\left(k_{i+1}^{2}-m^{2}\right)-\frac{1}{2}\left(k_{i-1}^{2}-m^{2}\right) \\
= & 0 .
\end{aligned}
$$

To show that the propagator requirement Eq. (9) is also satisfied, let $S=\{p, p+1, p+2, \ldots, q\}$, with $|S|:=q-$ $p+1 \leq n-2$. The latter condition is necessary to ensure that there are at least two lines in $S$ to merge to form a propagator somewhere. Since $a_{i i}=0$,

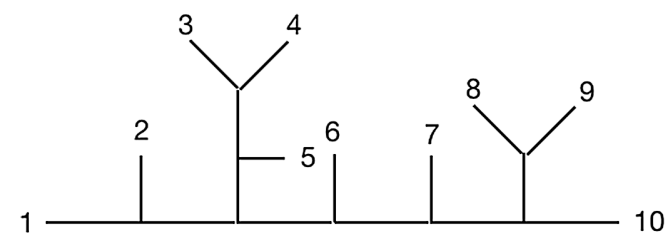

(a)

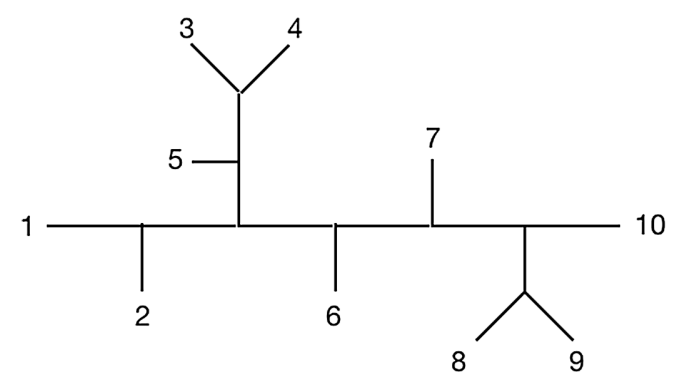

(c) $\sum_{i, j \in S} a_{i j}=\sum_{i \neq j ; i, j \in S} a_{i j}=2 \sum_{i=p}^{q-1} \sum_{j=i+1}^{q}\left(k_{i} \cdot k_{j}+\rho_{i j}-\frac{1}{2} \mu_{i j}\right)$.

For $j>i$, the only nonzero $\rho_{i j}$ 's are for $j=i+1$ and $j=i+2$. Hence

$$
\begin{aligned}
& 2 \sum_{i=p}^{q-1} \sum_{j=i+1}^{q}\left(k_{i} \cdot k_{j}+\rho_{i j}\right) \\
& =2 \sum_{i=p}^{q-1} \sum_{j=i+1}^{q} k_{i} \cdot k_{j}+2 \sum_{i=p}^{q-2}\left[\rho_{i, i+1}+\rho_{i, i+2}\right]-2 \rho_{q-2,+q-1} \\
& =2 \sum_{i=p}^{q-1} \sum_{j=i+1}^{q} k_{i} \cdot k_{j}+\sum_{i=p}^{q-2}\left[\left(k_{i}^{2}+k_{i+1}^{2}\right)-k_{i+1}^{2}\right] \\
& \quad+\left(k_{q-1}^{2}+k_{q}^{2}\right) \\
& =\left(\sum_{i=p}^{q} k_{i}\right)^{2}=k_{S}^{2}
\end{aligned}
$$

and

$\sum_{i=p}^{q-1} \sum_{j=i+1}^{q} \mu_{i j}=\sum_{i=q}^{q-2}\left[\mu_{i, i+1}+\mu_{i, i+2}\right]-\mu_{q-2, q-1}=m^{2}$.

Thus the propagator condition Eq. (9) is satisfied with $m_{S}^{2}=m^{2}$.

\section{E. Connection between $\sigma_{(\alpha)}$ and Feynman diagrams}

Given a planar tree diagram such as Fig. 1(a) whose external lines are ordered clockwise and cyclically

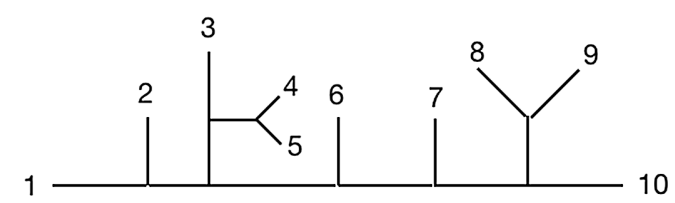

(b)

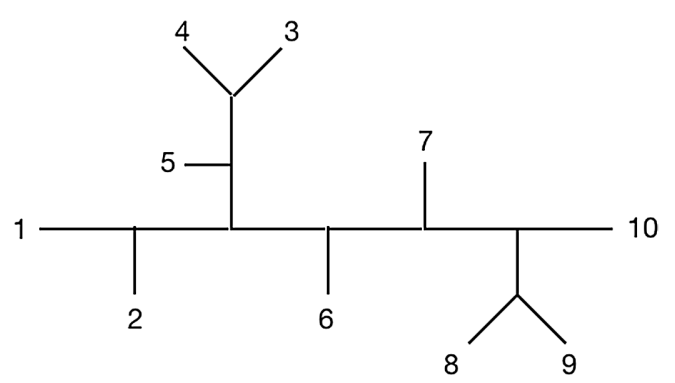

(d)

FIG. 1. $\sigma_{(\alpha)}$ and propagators. 
according to $(12 \cdots n)$, there are several techniques [3,7-11,19] to determine the appropriate $\sigma_{(\alpha)}$ that yields the diagram. In this subsection we review how to do that following the approach of $[11,19]$.

To that end, note that there are many ways to redraw a diagram by flipping its external lines. For Fig. 1(a), one of them is Fig. 1(c), another one is Fig. 1(d), and there are many others. The flipped diagrams possess the same propagators as the original diagram, hence the same scalar amplitude, but their external lines are cyclically ordered in different ways. One of them would give the $\sigma_{(\alpha)}$ we are after.

To find out which, we have to know the general relation between propagators and $\sigma_{(\alpha)}$. For planar diagrams, propagators come from a merging of consecutive external lines. It turns out that consecutive external lines labeled by $\alpha_{u}, \alpha_{u+1}, \ldots, \alpha_{u+m}$ would merge into a propagator if and only if they form a permutation of $m$ consecutive numbers $p, p+1, \ldots, p+m$. When that happens we will put a square bracket around them, as in $\left[\alpha_{u} \alpha_{u+1} \cdots \alpha_{u+m}\right]$. A Feynman tree diagram has $n-3$ propagators, so $(\alpha)$ must contain $n-3$ such compatible square brackets. Two brackets are compatible if either they do not overlap or one is completely inside another.

If there is only one way to partition $(\alpha)$ into such compatible square brackets, then this $\sigma_{(\alpha)}$ would give rise to one Feynman diagram. If there is more than one way to partition $(\alpha)$, then the amplitude would receive contributions from several Feynman diagrams, each corresponding to one such partition.

For example, the ordering $\alpha=(6215347(10) 98)$ in Fig. 1(c) can be partitioned only one way, into ([6[[21] $\times$ $[5[34]]]] 7[(10)[98]])$, so this is the right $\alpha$ for the single diagram Fig. 1(a). However, $\alpha=(6215437(10) 98)$ in Fig. 1(d) has two compatible partitions, $([6[[21][5[43]]]] \times$ $7[(10)[98]])$ and $([6[[21][[54] 3]]] 7[(10)[98]])$, so that $\sigma_{(\alpha)}$ gives rise to both Fig. 1(a) and Fig. 1(b), when the external lines are ordered according to $(12 \cdots n)$. More complicated $\alpha$ can give rise to more allowed partitions and more Feynman diagrams. In particular, the identity permutation $\sigma_{(\alpha)}=\sigma_{(12 \cdots n)}$ yields a sum of all Feynman tree diagrams.

In summary, given a single diagram, the appropriate $\sigma_{(\alpha)}$ is given by that flipping of external lines that produces a single compatible partition for $(\alpha)$. A different flipping may result in a sum of several diagrams, and $\sigma_{(\alpha)}=\sigma_{(12 \cdots n)}$ would give rise to a sum of all Feynman diagrams.

This rule works not only for the $\phi^{3}$ theory but also for all the other theories to be discussed in the following sections.

It would be useful to know the origin of this rule [11,19], so that it can be generalized to the situation in Sec. VII. The $(n-3)$-fold integration in Eq. (7) is taken over every $\sigma_{i}$, except $\sigma_{r}, \sigma_{s}, \sigma_{t}$. Contribution to the integral comes from simple poles in the factor $1 / \sigma_{(\alpha)}$, located when the $\sigma_{i}$ 's inside a square bracket coincide with one another. When the numbers inside a square bracket are permutations of a consecutive subset of $(12 \cdots n)$, a pole also occurs in the other factor $1 / \sigma_{(12 \cdots n)}$ of Eq. (8), but this other pole is compensated by a zero found in the factor $1 / \prod_{i \neq r, s, t} f_{i}$, leaving behind a propagator as the residue. In this way the $(n-3)$ integrations in Eq. (7) give rise to $(n-3)$ propagators that make up a Feynman diagram.

\section{F. Beyond the $\phi^{3}$ theory}

If we decompose $a_{i j}$ in the form of Eq. (12), with $\rho_{i j}$ given by Eq. (13), then Eq. (14) and Eq. (16) show that the symmetry condition $a_{i j}=a_{j i}$, covariant condition Eq. (2), and the propagator condition Eq. (9) are already satisfied by the momentum part of $a_{i j}$. That leaves only the mass part $\mu_{i j}$, which must satisfy

$$
\begin{gathered}
\mu_{i j}=\mu_{j i} \quad(\text { symmetry condition), } \\
\sum_{j \neq i} \mu_{i j}=0 \quad \text { (covariant condition), } \\
\frac{1}{2} \sum_{i \neq j, i, j \in S} \mu_{i j}=m_{S}^{2}, \\
|S| \leq n-2 \quad \text { (propagator condition), }
\end{gathered}
$$

where $|S|$ is the number of lines in $S$. Since $m_{S}^{2}$ depends on all the lines in $S, \mu_{i j}$ must depend on the particle nature of $i$, $j$, and all the lines in between. To exhibit such a dependence explicitly, we shall denote $\mu_{i j}$ by $\left(a_{i} a_{i+1} \cdots a_{j}\right)$ when $j>i$, where $a$ is a particle identification symbol: $a_{i}$ specifies the particle of line $i, a_{j}$ the particle of line $j$, and the remaining $a$ 's specify the particle identity of lines in between. The inequality $j>i$ means that line $j$ is downstream from line $i$ when external lines are ordered cyclically in the clockwise direction. It does not necessarily mean that the number $j$ is larger than the number $i$ because of the cyclic nature of the external lines.

Among the three terms of $a_{i j}$ in Eq. (12), the $k_{i} \cdot k_{j}$ term is the simplest because it depends only on lines $i$ and $j$. The $\rho_{i j}$ term is more complicated because it can depend on the lines between $i$ and $j$, e.g., $\rho_{i, i \pm 2}=\frac{1}{2} k_{i \pm 1}^{2}$, but it becomes zero when $j$ and $i$ are more than two lines apart. The last term $\mu_{i j}$ is the most complicated because not only does it depend on all lines between $i$ and $j$, it may also be nonzero no matter how far apart lines $i$ and $j$ are. Moreover, it also depends on the precise ordering and identity of the particles in between.

Since kinematics are universal, the $k_{i} \cdot k_{j}$ and $\rho_{i j}$ terms are the same in all theories. Thus it is the $\mu_{i j}$ term that tells theories apart. However, even for the same theory, different ordered sets of external lines may give rise to different $\mu_{i j}$ 's. For example, in the Yukawa theory to be discussed in the next section, where solid and dotted lines represent scalar nucleons and pions, respectively, the amplitude in Fig. 2(a) and the amplitude in Fig. 2(b) may have different sets of $\mu_{i j}$. 


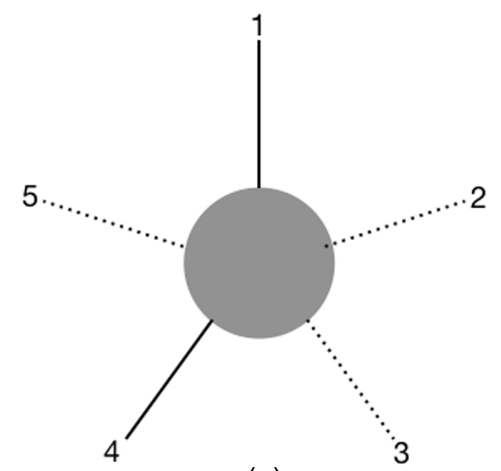

(a)

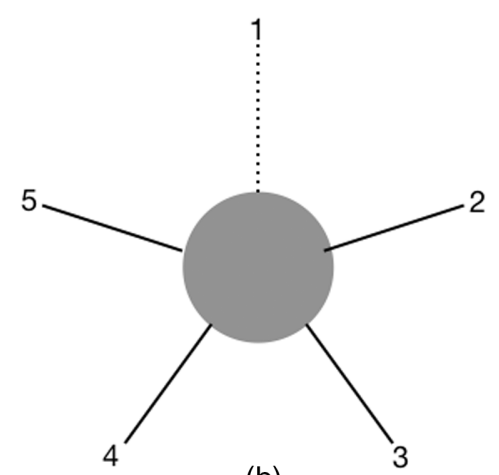

(b)

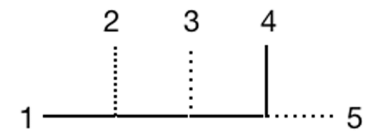

(a1)

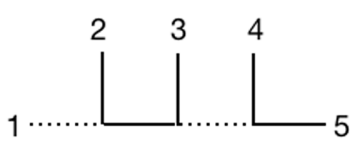

(b1)

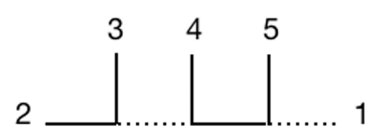

(b3)

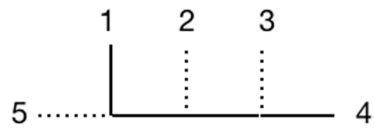

(a2)

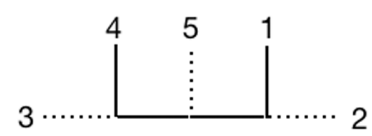

(a3)

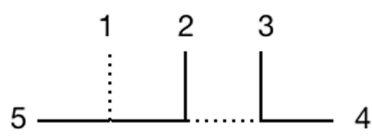

(b2)

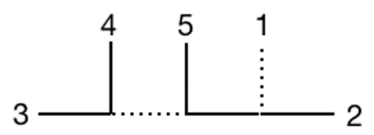

(b4)

FIG. 2. Two tree amplitudes for Yukawa interaction. Solid lines are nucleons, and dotted lines are pions.

Diagrams (a1), (a2), (a3) are the tree diagrams contained in Fig. 2(a), and (b1), (b2), (b3), (b4) are the tree diagrams contained in Fig. 2(b). $\mu_{i j}$ for (a1), (a2), (a3) are the same, but that may not be the same as the $\mu_{i j}$ for (b1), (b2), (b3), and (b4).

Let us delve a bit more into the details on how Eq. (9) can be used to compute $\mu_{i j}$. If $S$ consists of consecutive lines from $p$ to $q$, then we will write the left-hand side of Eq. (20) as $\left\langle a_{p} a_{p+1} \cdots a_{q}\right\rangle$. With that notation, Eq. (20) can be written as

$$
\begin{aligned}
m_{S}^{2} & =\left\langle a_{p} a_{p+1} \cdots a_{q}\right\rangle=\sum_{i=p}^{q-1} \sum_{j=i+1}^{q} \mu_{i j} \\
& =\sum_{i=p}^{q-1} \sum_{j=i+1}^{q}\left(a_{i} a_{i+1} a_{i+2} \cdots a_{j}\right) .
\end{aligned}
$$

This allows $\mu_{i j}$ to be computed by four combinations of $m_{S}^{2}$ using the formula

$$
\begin{aligned}
\mu_{i j}= & \left(a_{i} a_{i+1} \cdots a_{j-1} a_{j}\right)=\left\langle a_{i} a_{i+1} \cdots a_{j-1} a_{j}\right\rangle \\
& -\left\langle a_{i+1} \cdots a_{j-1} a_{j}\right\rangle-\left\langle a_{i} a_{i+1} \cdots a_{j-1}\right\rangle \\
& +\left\langle a_{i+1} \cdots a_{j-1}\right\rangle \quad(j>i),
\end{aligned}
$$

if $n-2 \geq|S|=q-p+1 \geq 4$. For smaller $|S|$, the appropriate formulas are

$$
\begin{aligned}
\left(a_{p} a_{p+1}\right) & =\left\langle a_{p} a_{p+1}\right\rangle, \\
\left(a_{p} a_{p+1} a_{p+2}\right) & =\left\langle a_{p} a_{p+1} a_{p+2}\right\rangle-\left\langle a_{p} a_{p+1}\right\rangle-\left\langle a_{p+1} a_{p+2}\right\rangle .
\end{aligned}
$$

To use these formulas to determine $\mu_{i j}$, we must know what $m_{S}^{2}$ is. That depends on the propagator, which in turn depends on the nature of interaction and the particle content of the external lines in $S$. More specifically, an interaction determines a set of "topological requirements," which in turn fixes $m_{S}^{2}$. It will become clear that $m_{S}^{2}$ depends only on the particle content of $S$, but never on their ordering, so $\left\langle a_{p} a_{p+1} \cdots a_{q}\right\rangle$ is permutation invariant in the symbols. However, $\left(a_{p} a_{p+1} \cdots a_{q}\right)$ does depend on how the lines are ordered. Nevertheless, it follows from Eq. (22) and Eq. (23) that the reversal relation

$$
\left(a_{p} a_{p+1} \cdots a_{q-1} a_{q}\right)=\left(a_{q} a_{q-1} \cdots a_{p+1} a_{p}\right)
$$

is always valid.

\section{G. Complementary, symmetry, and covariant conditions}

It will be shown in this subsection that once the complementary condition Eq. (10) is obeyed, the symmetry condition Eq. (18) and the covariant condition Eq. (19) are automatically satisfied. For that reason, the important thing 
to check for each theory is whether the complementary condition is fulfilled. In using the propagator condition to determine $\mu_{i j}$, it turns out that often unknown parameters have to be introduced. Forcing the complementary condition to be valid would then determine some or all of these parameters.

\section{Symmetry condition}

If $\mu_{i j}$ is computed using Eq. (22) for $j>i$, then $\mu_{j i}$ should be computed using Eq. (22) for $i>j$. Note that these two inequalities are not mutually contradictory because they simply mean that the second index in each case is downstream from the first index in a clockwise direction. It says nothing about the numerical sizes of $i$ vs $j$. With that in mind, we have

$$
\begin{aligned}
\mu_{i j}= & \langle i, i+1, \ldots, j-1, j\rangle-\langle i+1, \ldots, j-1, j\rangle \\
& -\langle i, i+1, \ldots, j-1\rangle+\langle i+1, \ldots, j-1\rangle, \\
\mu_{j i}= & \langle j, j+1, \ldots, i-1, i\rangle-\langle j+1, \ldots, i-1, i\rangle \\
& -\langle j, j+1, \ldots, i-1\rangle+\langle j+1, \ldots, i-1\rangle .
\end{aligned}
$$

Each of the four terms in $\mu_{i j}$ contains lines that are complementary to lines in one term of $\mu_{j i}$. For example, the complement of the first term in $\mu_{i j}$ is the last term of $\mu_{j i}$. Using the complementary condition $m_{S}^{2}=m_{\bar{S}}^{2}$, it follows that $\mu_{i j}=\mu_{j i}$.

\section{Covariant condition}

For the covariant condition Eq. (19) from the propagator condition, it is important to note that Eq. (20) is valid only for $|S| \leq n-2$. With that caveat and using the symmetry condition for the last two terms,

$$
\begin{aligned}
\sum_{j \neq i} \mu_{i j}= & \sum_{j=i+1}^{i-3} \mu_{i j}+\mu_{i-2, i}+\mu_{i-1, i} \\
= & \langle i, i+1, \ldots, i-3\rangle-\langle i+1, \ldots, i-3\rangle \\
& +\langle i-2, i-1, i\rangle-\langle i-2, i-1\rangle .
\end{aligned}
$$

The lines in the first and last terms are complementary, and the lines in the second and third terms are also complementary. Thus Eq. (10) ensures the right-hand side of the above equation to vanish.

\section{H. Uniqueness of $a_{i j}$}

$a_{i j}$ given by Eq. (12), Eq. (20), and Eq. (22) is unique if we use these expressions on all possible propagators in all Feynman diagrams of a given ordering of external lines. If we only want to find an $a_{i j}$ that yields a single diagram, then there are many ways to choose it, because the propagator condition Eq. (9) has to be satisfied only by $n-3$ sets $S$, instead of all the consecutive sets.
The same remark holds for all the other theories to be considered in later sections.

\section{YUKAWA THEORY AND THE $\phi^{4}$ THEORY}

\section{A. Topological and propagator conditions}

Consider a scalar nucleon field $\phi$ with mass $m_{1}$ and a pion field $\phi^{\prime}$ with mass $m_{0}$, coupled via a Yukawa interaction $\phi^{2} \phi^{\prime}$. Each vertex has two nucleon lines and one pion line; an $n$-point tree amplitude has $n-3$ propagators. If $E, E^{\prime}$ is the number of external lines of nucleons and pions, and $I, I^{\prime}$ is their number of internal lines, then

$$
\begin{aligned}
& E+2 I=2\left(E^{\prime}+2 I^{\prime}\right), \\
& E+E^{\prime}=n=I+I^{\prime}+3 .
\end{aligned}
$$

Solving for $I$ and $I^{\prime}$, the solution is

$$
\begin{aligned}
I & =\frac{1}{2} E+E^{\prime}-2, \\
I^{\prime} & =\frac{1}{2} E-1 .
\end{aligned}
$$

In order for $I, I^{\prime}$ to be non-negative integers, $E$ must be an even integer $\geq 2$. When $E=2$, then we must have $E^{\prime} \geq 1$. This topological requirement applies not only to the whole Feynman diagram, it also applies to a subdiagram consisting of a propagator and all its corresponding external lines. It is the basis from which $\mu_{i j}$ for the Yukawa theory is determined.

If the set $S$ of consecutive external lines consists of an even number of nucleons, then the propagator line must be a pion so $m_{S}^{2}=m_{0}^{2}$. If the set consists of an odd number of nucleons, then the propagator line must be a nucleon so $m_{S}^{2}=m_{1}^{2}$. If the set consists of only pions, then no propagator can be formed, so $m_{S}^{2}$ is undetermined. We shall assign it a value $M^{2}$ where $M$ is so far arbitrary. To summarize, using $N(S)$ to denote the number of nucleons in the set $S$, then

$$
\begin{aligned}
m_{S}^{2} & =m_{0}^{2} \quad(N(S)=\text { even } \neq 0), \\
& =M^{2} \quad(N(S)=0), \\
& =m_{1}^{2} \quad(N(S)=\text { odd }) .
\end{aligned}
$$

\section{B. Complementary condition}

In order to satisfy the complementary condition $m_{S}^{2}=$ $m_{\bar{S}}^{2}$ of Eq. (10), we need to set $M^{2}=m_{0}^{2}$. This is so because the nucleon number of the whole diagram is even, so $(-)^{N(S)}=(-)^{N(\bar{S})}$. In particular, if $N(S)$ is even, then it is guaranteed that $N(\bar{S})$ is even, but depending on the diagram and the configuration of external lines, it may or may not be zero. So in order for Eq. (10) to be satisfied for all conceivable sets $S$, we must let $M^{2}=m_{0}^{2}$ in Eq. (29). 


\section{Illustrative examples}

Let $a=0$ denote a pion and $a=1$ a nucleon. Using Eq. (22), Eq. (23), and Eq. (29) with $M^{2}=m_{0}^{2}$, we get

$$
\begin{aligned}
(00) & =\{00\}=m_{0}^{2}, \quad(01)=(10)=\{01\}=m_{1}^{2}, \quad(11)=\{11\}=m_{0}^{2}, \\
(011) & =(110)=\{110\}-\{01\}-\{11\}=-m_{1}^{2}, \quad(000)=\{000\}-2\{00\}=-m_{0}^{2}, \\
(100) & =(001)=\{100\}-\{10\}-\{00\}=-m_{0}^{2}, \quad(101)=\{101\}-\{10\}-\{01\}=m_{0}^{2}-2 m_{1}^{2}, \\
(111) & =\{111\}-2\{11\}=m_{1}^{2}-2 m_{0}^{2} .
\end{aligned}
$$

For $|S| \geq 4, \mu_{i j}$ can be written in the form

$$
(a V b)=\langle a V b\rangle-\langle a V\rangle-\langle V b\rangle+\langle V\rangle,
$$

where $a, b$, and the members of the subset $V$ are 0 's or 1's. Using Eq. (29), it follows that $(a V b)=0$ if either $a$ or $b$ is 0 , because the four terms cancel pairwise. The only nonzero $\mu_{i j}$ is of the form $(1 V 1)$, and it is equal to $2\left(m_{0}^{2}-m_{1}^{2}\right)$ or $2\left(m_{1}^{2}-m_{0}^{2}\right)$ depending on whether $V$ contains an even number of nucleons or an odd number of nucleons.

These results are summarized in the following equation, where $a$ and $b$ are either 0 or 1 , and $N(V)$ stands for the number of nucleons in the subset $V$ :

$$
\begin{aligned}
(00) & =(11)=m_{0}^{2}, \quad(01)=(10)=m_{1}^{2}, \quad(011)=(110)=-m_{1}^{2}, \\
(000) & =(100)=(001)=-m_{0}^{2}, \quad(101)=m_{0}^{2}-2 m_{1}^{2}, \quad(111)=m_{1}^{2}-2 m_{0}^{2}, \\
(0 V b) & =(a V 0)=0 \quad(|V| \geq 2), \\
(1 V 1) & =2\left(m_{0}^{2}-m_{1}^{2}\right)(N(V)=\text { even }), \quad(1 V 1)=2\left(m_{1}^{2}-m_{0}^{2}\right)(N(V)=\text { odd }) .
\end{aligned}
$$

\section{Elastic nucleon-nucleon and pion-nucleon scatterings}

Let us apply Eq. (7) to the scattering diagrams shown in Fig. 3. By choosing the Möbius constant lines $r, s, t$ to be $2,3,4$, only $f_{1}$ and $\mu_{1 j}$ enter into Eq. (7).

Recall that Eq. (21) can be used to compute $\mu_{i j}$ only when $j-i+1 \leq n-2$, because it requires at least two external lines to merge into a propagator. Thus for $n=4$, Eq. (31) can be used only when $j=i+1$. In particular, for $i=1$, only $\mu_{12}$ can be so calculated, though $\mu_{14}$ can also be calculated using the symmetry condition $\mu_{14}=$ $\mu_{41}=\mu_{45}$. That leaves $\mu_{13}$, which cannot be calculated from Eq. (21), but it can be calculated from Eq. (19) as $\mu_{13}=-\left(\mu_{12}+\mu_{14}\right)$.

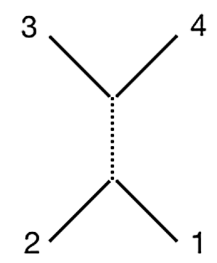

(a)

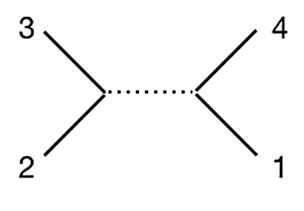

(b)

For Fig. 3(a) and Fig. 3(b), using Eq. (31),

$$
\begin{aligned}
& \mu_{12}=(11)=m_{0}^{2}, \quad \mu_{14}=\mu_{41}=(11)=m_{0}^{2}, \\
& \mu_{13}=-\left(\mu_{12}+\mu_{14}\right)=-2 m_{0}^{2} .
\end{aligned}
$$

For Fig. 3(c) and Fig. 3(d),

$$
\begin{aligned}
& \mu_{12}=(01)=m_{1}^{2}, \quad \mu_{14}=\mu_{41}=(10)=m_{1}^{2}, \\
& m_{13}=-\left(\mu_{12}+\mu_{14}\right)=-2 m_{1}^{2} .
\end{aligned}
$$

The $\sigma_{(\alpha)}$ factor is $\sigma_{(2134)}$ for Fig. 1(a) and Fig. 1(c), and is $\sigma_{(4132)}$ for Fig. 1(b) and Fig. 1(d). Thus the inverse propagators are $2 a_{12}=2 k_{1} \cdot k_{2}+k_{1}^{2}+k_{2}^{2}-\mu_{12}=\left(k_{1}+k_{2}\right)^{2}-m_{0}^{2}$

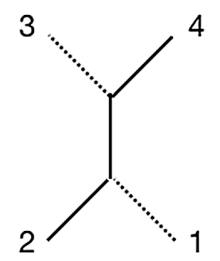

(c)

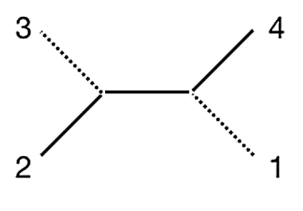

(d)

FIG. 3. Elastic nucleon-nucleon and pion-nucleon scattering diagrams. Solid lines are nucleons and dotted lines are pions. 
for Fig. 3(a), $2 a_{14}=2 k_{1} \cdot k_{4}+k_{1}^{2}+k_{4}^{2}-\mu_{14}=\left(k_{1}+k_{4}\right)^{2}-$ $m_{0}^{2}$ for Fig. 3(b), $2 a_{12}=2 k_{1} \cdot k_{2}+k_{1}^{2}+k_{2}^{2}-\mu_{12}=\left(k_{1}+\right.$ $\left.k_{2}\right)^{2}-m_{1}^{2}$ for Fig. 3(c), and $2 a_{14}=2 k_{1} \cdot k_{4}+k_{1}^{2}+k_{4}^{2}-$ $\mu_{14}=\left(k_{1}+k_{4}\right)^{2}-m_{1}^{2}$ for Fig. 3(d), all agreeing with what the diagrams indicate.

Note that $\mu_{13}$ and $\mu_{14}$ are not needed for Figs. 3(a) and 3(c), and $\mu_{13}$ and $\mu_{12}$ are not needed for Figs. 3(b) and 3(d), so they could have been something else without affecting the propagator. See Sec. II H for more discussions on this point.

Note also that we can get Fig. 1(a) and Fig. 1(b) together, also Fig. 1(c) plus Fig. 1(d) at the same time, by choosing $\sigma_{(\alpha)}=\sigma_{(1234)}$.

$$
\text { 2. } N+N \rightarrow N+N+\pi+\pi
$$

There are many diagrams for the emission of two pions. For the purpose of illustration we consider only Fig. 4.

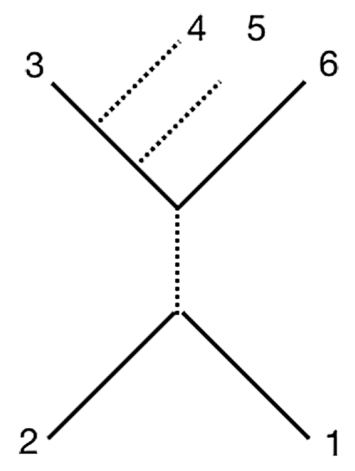

FIG. 4. A nucleon-nucleon scattering diagram with the emission of two pions.

In this case, by fixing the Möbius constant lines $r, s, t$ to be $2,5,6$, only $f_{1}, f_{3}, f_{4}$ appear in Eq. (7). The corresponding mass terms calculated from Eq. (31) are

$$
\begin{aligned}
& \mu_{12}=(11)=m_{0}^{2}, \quad \mu_{13}=(111)=-2 m_{0}^{2}+m_{1}^{2}, \quad \mu_{14}=(1110)=0, \\
& \mu_{15}=\mu_{51}=(011)=-m_{1}^{2}, \quad \mu_{16}=\mu_{61}=(11)=m_{0}^{2}, \\
& \mu_{31}=\mu_{13}=-2 m_{0}^{2}+m_{1}^{2}, \quad \mu_{32}=\mu_{23}=(11)=m_{0}^{2}, \quad \mu_{34}=(10)=m_{1}^{2}, \\
& \mu_{35}=(100)=-m_{0}^{2}, \quad \mu_{36}=(1001)=2\left(m_{0}^{2}-m_{1}^{2}\right), \\
& \mu_{41}=\mu_{14}=0, \quad \mu_{42}=\mu_{24}=(110)=-m_{1}^{2}, \quad \mu_{43}=\mu_{34}=m_{1}^{2}, \\
& \mu_{45}=(00)=m_{0}^{2}, \quad \mu_{46}=(001)=-m_{0}^{2}, \\
& \sigma_{(\alpha)}=\sigma_{(215346)} .
\end{aligned}
$$

It can be directly checked from these relations that the covariant requirement $\sum_{j \neq i} \mu_{i j}=0$ is satisfied for $i=1,3,4$.

The three inverse propagators

$$
\begin{aligned}
2 a_{12} & =\left(k_{1}+k_{2}\right)^{2}-\mu_{12}=\left(k_{1}+k_{2}\right)^{2}-m_{0}^{2}, \\
2 a_{34} & =\left(k_{3}+k_{4}\right)^{2}-\mu_{34}=\left(k_{3}+k_{4}\right)^{2}-m_{1}^{2}, \\
2\left(a_{34}+a_{35}+a_{45}\right) & =\left(k_{3}+k_{4}+k_{5}\right)^{2}-\left(\mu_{34}+\mu_{35}+\mu_{45}\right)=\left(k_{3}+k_{4}+k_{5}\right)^{2}-m_{1}^{2},
\end{aligned}
$$

are as shown in the diagram.

\section{D. $\sigma_{(\alpha)}$ and sum over diagrams}

So far we have discussed how to choose $\sigma_{(\alpha)}$ and $f_{i}$ in Eq. (7) to compute the amplitude of a single tree diagram in the Yukawa theory. For the $\phi^{3}$ theory, Sec. II E shows how $\sigma_{(\alpha)}$ can also be chosen to yield a sum of several, or all, Feynman diagrams in the amplitude. Since the method of choosing $\sigma_{(\alpha)}$ has nothing to do with $f_{i}$, it is expected to be valid for the Yukawa theory as well. This is indeed so, but with one caveat.

Recall the method consists of partitioning $(\alpha)$ into $n-3$ compatible square brackets, each representing a propagator in a Feynman diagram. For the Yukawa theory, this is still true as long as we avoid those partitions containing square brackets with only pion lines. We must exclude those because pions alone cannot merge into a propagator, which is why we assign an arbitrary constant $M^{2}$ to that case in Eq. (29). If it were not for the complementary condition which forces $M^{2}$ to be $m_{0}^{2}$, even those diagrams need not be avoided because we could have set $M^{2}=\infty$ to get rid of them at the end. As it is, $M^{2}=m_{0}^{2}$, but this is not the propagator mass of several pions, because such a set does not even produce a propagator. To get things right, we must eliminate those phantom propagators by hand, by excluding the partitions containing pure pion square brackets. As long as that is followed, everything else is the same as in the $\phi^{3}$ theory.

For example, the particles in the six external lines of Fig. 4 are of the type (111001), with lines 4 and 5 being two 


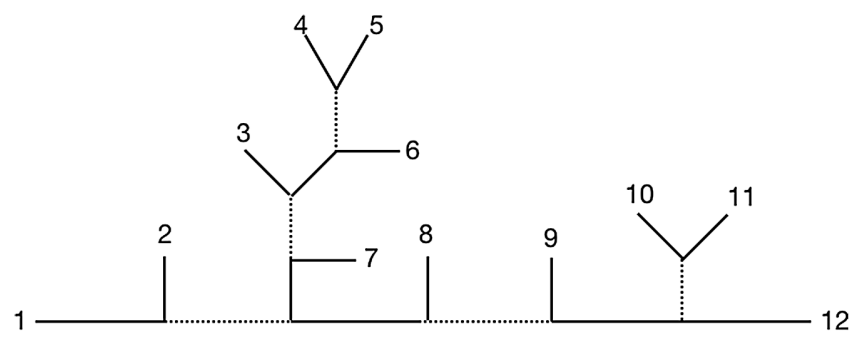

(a)

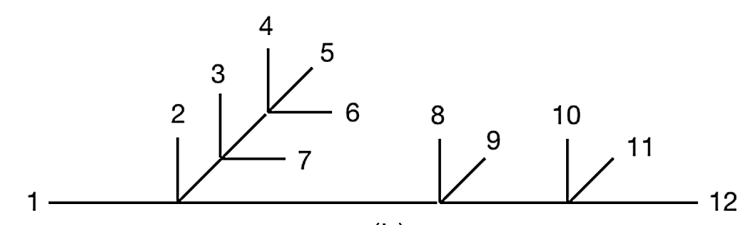

(b)

FIG. 5. A diagram with $\phi^{4}$ vertices such as (b) can be obtained from a Yukawa diagram such as (a) by setting the pion mass to be infinity.

consecutive pion lines. By setting $\sigma_{(\alpha)}=\sigma_{(123456)}$, one would have included diagrams containing a two-pion phantom propagator and produced a wrong result, unless we exclude by hand partitions that have pure pion square brackets. However, if the fifth pion line were attached to the downstream side of the next nucleon, so that the particle content becomes (111010), then there are no consecutive pion sets so $\sigma_{(\alpha)}=\sigma_{(123456)}$ for that kind of $\mu_{i j}$ would produce the correct sum of all Feynman tree diagrams with those particle contents.

\section{E. The $\phi^{3}$ limit}

By setting $m_{0}=m_{1}=m$ we recover the $\phi^{3}$ theory from the Yukawa theory. In that limit, it follows from Eq. (31) that $\mu_{i, i \pm 1}=m^{2}, \mu_{i, i \pm 2}=-m^{2}$, and $\mu_{i j}=0$ for $|j-i|>2$, which is what it should be according to Eq. (13).

\section{F. The $\phi^{4}$ theory}

In the $m_{0} \rightarrow \infty$ limit, a multinucleon tree diagram such as Fig. 5(a) becomes a $\phi^{4}$ diagram such as Fig. 5(b), provided the coupling constant is suitably scaled by a power of $m_{0}$. However, it is important to remember that the $m_{0} \rightarrow \infty$ limit should be taken after the integral Eq. (7) is carried out.

\section{MULTIPLE PARTICLE YUKAWA THEORY}

The Yukawa theory of the last section can be generalized to include several kinds of scalar nucleons and pions, all with different masses. In order not to make the model and the notation too complicated, we consider here only two kinds of nucleons, with masses $m_{1}$ and $m_{2}$, respectively. Each kind can interact with pions of mass $m_{0}$ via the Yukawa interaction $\phi_{1}^{2} \phi^{\prime}+\phi_{2}^{2} \phi^{\prime}$. For simplicity, we have also set both coupling constants equal to 1 .

\section{A. Propagator condition}

There must be an even number of external nucleons of each kind in a Feynman diagram, or a subdiagram with one propagator. Moreover, in the tree approximation, there can be no Feynman diagram with external pions alone. Let $N_{1}$, $N_{2}$ be the number of external lines for the two kinds of nucleons, then $m_{S}^{2}$ for a set $S$ of consecutive external lines can be determined as before to be

$$
\begin{aligned}
m_{S}^{2} & =m_{0}^{2} \quad \text { if }\left(N_{1}, N_{2}\right)=(\text { even, even }) \neq(0,0), \\
& =M^{2} \quad \text { if }\left(N_{1}, N_{2}\right)=(0,0), \\
& =m_{1}^{2} \quad \text { if }\left(N_{1}, N_{2}\right)=(\text { odd, even }) \\
& =m_{2}^{2} \quad \text { if }\left(N_{1}, N_{2}\right)=(\text { even, odd }) \\
& =\bar{M}^{2} \quad \text { if }\left(N_{1}, N_{2}\right)=(\text { odd, odd }) .
\end{aligned}
$$

Both $M^{2}, \bar{M}^{2}$ are arbitrary parameters because these sets of external lines cannot merge into a propagator.

\section{B. Complementary condition}

Using the fact that the whole diagram must be an even number of nucleons of each kind, one can conclude that $(-)^{N_{1}(S)}=(-)^{N_{1}(\bar{S})}$ and $(-)^{N_{2}(S)}=(-)^{N_{2}(\bar{S})}$. From Eq. (36), it follows that the complementary condition Eq. (10) is satisfied if and only if $M^{2}=m_{0}^{2}$. Note that the unknown parameter $\bar{M}^{2}$ remains undetermined, so it must not enter into any propagator in any diagram.

\section{Illustrative example}

As before, the propagator condition Eq. (21) can be used to compute $\mu_{i j}$. Here are some results for small $|S|$. The reversal condition Eq. (24) can get us some more. Let $a=0,1,2$ denote a pion, the first kind of nucleon, and the second kind of nucleon. By setting $M^{2}=m_{0}^{2}$ and using Eq. (22) and Eq. (23), one gets, for $|S|=2$,

$$
\begin{aligned}
& (00)=m_{0}^{2}, \quad(12)=\bar{M}^{2}, \\
& (11)=(22)=m_{0}^{2}, \quad(01)=m_{1}^{2}, \quad(02)=m_{2}^{2} .
\end{aligned}
$$

For $|S|=3$, 


$$
\begin{aligned}
& (000)=-m_{0}^{2}, \quad(111)=\langle 111\rangle-\langle 11\rangle-\langle 11\rangle=m_{1}^{2}-2 m_{0}^{2}, \quad(222)=m_{2}^{2}-2 m_{0}^{2}, \\
& (012)=\langle 012\rangle-\langle 01\rangle-\langle 12\rangle=-m_{1}^{2}, \quad(021)=\langle 021\rangle-\langle 02\rangle-\langle 21\rangle=-m_{2}^{2}, \\
& (011)=\langle 011\rangle-\langle 01\rangle-\langle 11\rangle=-m_{1}^{2}, \quad(022)=\langle 022\rangle-\langle 02\rangle-\langle 22\rangle=-m_{2}^{2}, \\
& (102)=\langle 102\rangle-\langle 10\rangle-\langle 02\rangle=\bar{M}^{2}-m_{1}^{2}-m_{2}^{2}, \quad(101)=\langle 101\rangle-\langle 10\rangle-\langle 01\rangle=m_{0}^{2}-2 m_{1}^{2}, \\
& (202)=\langle 202\rangle-\langle 20\rangle-\langle 02\rangle=m_{0}^{2}-2 m_{2}^{2}, \quad(001)=\langle 001\rangle-\langle 00\rangle-\langle 01\rangle=-m_{0}^{2}, \\
& (002)=\langle 002\rangle-\langle 00\rangle-\langle 02\rangle=-m_{0}^{2}, \quad(010)=\langle 010\rangle-\langle 01\rangle-\langle 10\rangle=-m_{1}^{2}, \\
& (020)=-m_{2}^{2}, \quad(112)=\langle 112\rangle-\langle 11\rangle-\langle 12\rangle=m_{2}^{2}-m_{0}^{2}-\bar{M}^{2}, \\
& (221)=m_{1}^{2}-m_{0}^{2}-\bar{M}^{2}, \quad(121)=\langle 121\rangle-\langle 12\rangle-\langle 21\rangle=m_{2}^{2}-2 \bar{M}^{2}, \\
& (212)=m_{1}^{2}-2 \bar{M}^{2} .
\end{aligned}
$$

For $|S|=4$, only a few results are listed below for illustration, but clearly we can compute all the results for every $|S|$ :

$$
\begin{aligned}
& (1120)=\langle 1120\rangle-\langle 112\rangle-\langle 120\rangle+\langle 12\rangle=0, \\
& (1202)=\langle 1202\rangle-\langle 120\rangle-\langle 202\rangle+\langle 20\rangle=m_{1}^{2}-\bar{M}^{2}-m_{0}^{2}+m_{2}^{2}, \\
& (2020)=\langle 2020\rangle-\langle 202\rangle-\langle 020\rangle+\langle 02\rangle=0 \\
& (0201)=\langle 0201\rangle-\langle 020\rangle-\langle 201\rangle+\langle 20\rangle=0
\end{aligned}
$$

By taking the Möbius constant lines $r, s, t$ to be lines 4, 5, 6 in Fig. 6, only $f_{1}, f_{2}, f_{3}$ enter into Eq. (7). The relevant $\mu_{i j}$ can be computed from Eq. (38) and Eq. (39) to be

$$
\begin{aligned}
& \mu_{12}=(11)=m_{0}^{2}, \quad \mu_{13}=(112)=m_{2}^{2}-m_{0}^{2}-\bar{M}^{2}, \quad \mu_{14}=(1120)=0, \\
& \mu_{15}=\mu_{51}=(201)=\bar{M}^{2}-m_{1}^{2}-m_{2}^{2}, \quad \mu_{16}=\mu_{61}=(01)=m_{1}^{2}, \\
& \mu_{21}=\mu_{12}=(11)=m_{0}^{2}, \quad \mu_{23}=(12)=\bar{M}^{2}, \quad \mu_{24}=(120)=-m_{2}^{2}, \\
& \mu_{25}==(1202)=m_{1}^{2}-\bar{M}^{2}-m_{0}^{2}+m_{2}^{2}, \quad \mu_{26}=\mu_{62}=(011)=-m_{1}^{2}, \\
& \mu_{31}=\mu_{13}=(112)=m_{2}^{2}-m_{0}^{2}-\bar{M}^{2}, \quad \mu_{32}=\mu_{23}=\bar{M}^{2}, \quad \mu_{34}=(20)=m_{2}^{2}, \\
& \mu_{35}=(202)=m_{0}^{2}-2 m_{2}^{2}, \quad \mu_{36}=(2020)=0 .
\end{aligned}
$$

Using these formulas, it can be explicitly checked that $\sum_{j \neq i} \mu_{i j}=0$ for $i=1,2,3$. The inverse propagators in Fig. 6 can be obtained from Eq. (9), Eq. (12), and Eq. (40) to be

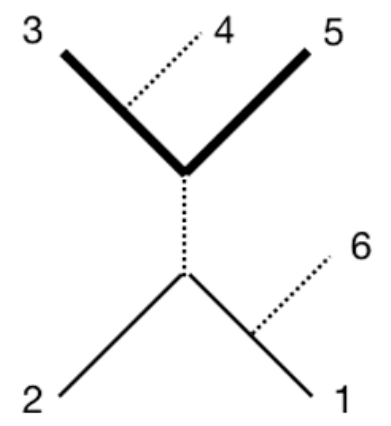

FIG. 6. Dotted lines are pions, and thin (thick) solid lines are nucleons 1 (2).

$$
\begin{aligned}
\left(k_{6}+k_{1}+k_{2}\right)^{2}-\left(\mu_{16}+\mu_{26}+\mu_{12}\right)= & \left(k_{6}+k_{1}+k_{2}\right)^{2} \\
& -m_{0}^{2}, \\
\left(k_{6}+k_{1}\right)^{2}-\mu_{16}= & \left(k_{6}+k_{1}\right)^{2}-m_{1}^{2}, \\
\left(k_{3}+k_{4}\right)^{2}-\mu_{34}= & \left(k_{3}+k_{4}\right)^{2}-m_{2}^{2} .
\end{aligned}
$$

Note that although the unknown parameter $\bar{M}^{2}$ may be contained in individual $\mu_{i j}$, they drop out in the covariant sum, and they also drop out in the propagator masses. For example, the propagator in the second nucleon can also be obtained also from lines $5,6,1,2$, so we should also have $m_{2}^{2}=\mu_{12}+\mu_{15}+\mu_{16}+\mu_{25}+\mu_{26}+\mu_{56}$. Except for $\mu_{56}$, which is $(20)=m_{2}^{2}$, all the other $\mu_{i j}$ already appear in Eq. (40). One can check although $\bar{M}^{2}$ appears in $\mu_{15}$ and $\mu_{25}$, it disappears from the sum, and the sum does add up to $m_{2}^{2}$. 


\section{V. $\phi_{1} \phi_{2} \phi_{3}$ THEORY}

\section{A. Topological rules for tree amplitudes}

For easy reference, we shall refer to the three fields as having three different "colors." Consider a connected tree diagram with $E_{a}\left(I_{a}\right)$ external (internal) lines of color $a$, and a total of $n$ external lines. Such a diagram has $n-3$ internal lines and $n-2$ vertices, and hence

$$
\begin{aligned}
E_{1}+E_{2}+E_{3} & =n=I_{1}+I_{2}+I_{3}+3, \\
E_{1}+2 I_{1} & =n-2=E_{2}+2 I_{2}=E_{3}+2 I_{3} .
\end{aligned}
$$

Given $E_{a}$, these rules lead to the solution

$$
\begin{aligned}
& I_{1}=\frac{1}{2}\left(E_{2}+E_{3}\right)-1, \\
& I_{2}=\frac{1}{2}\left(E_{1}+E_{3}\right)-1, \\
& I_{3}=\frac{1}{2}\left(E_{2}+E_{1}\right)-1 .
\end{aligned}
$$

In order for $I_{a}$ to be a non-negative integer, we must have $E_{1}, E_{2}, E_{3}$ to be either all odd or all even numbers. We must also have $E_{a}+E_{b} \geq 2$ for every pair $a, b$, thereby forbidding diagrams with only one color of external lines. These topological requirements are important for the determination of $\mu_{i j}$.

Figure 7 gives a concrete illustration of Eq. (42), with $n=10,\left[E_{1}, E_{2}, E_{3}\right]=[4,4,2]$, and $\left[I_{1}, I_{2}, I_{3}\right]=[2,2,3]$.

Using the topological requirements, a set $S$ of consecutive lines can be sorted into 10 different classes. $T_{0}, T_{1}, T_{2}, T_{3}$ are classes where the external lines in $S$ cannot merge into a propagator, and $S_{1}, S_{2}, S_{3}, S_{1}^{\prime}, S_{2}^{\prime}, S_{3}^{\prime}$ are classes that can. These classes are distinguished by the number of external lines $E_{a}^{S}$ of color $a$ in the class. The resulting subdiagram, consisting of the propagator and the lines in $S$, must itself satisfy the topological requirement. This requirement will be used to determine the propagator mass $m_{S}^{2}$.

$T_{0}$ is the class where $E_{a}^{S}$ are all even or all odd. $T_{a}$ $(a=1,2,3)$ is the class where all lines have the same color $a$. None of these classes can merge into a propagator, for otherwise the topological requirement would be violated for the subdiagram whatever color the propagator carries. With no propagator, $m_{S}^{2}$ is undetermined. We shall assign it to be $M_{0}^{2}$ for class $T_{0}$ and $M_{a}^{2}$ for class $T_{a}$. They may remain

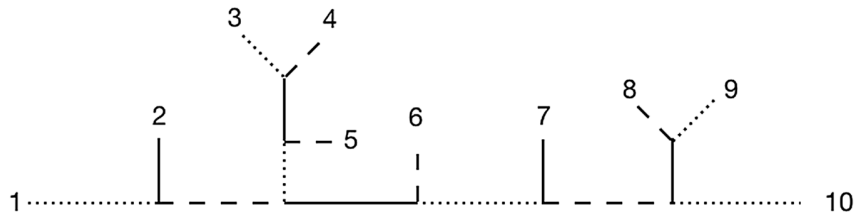

FIG. 7. A $\phi_{1} \phi_{2} \phi_{3}$ tree diagram, with $\phi_{1}, \phi_{2}, \phi_{3}$ depicted, respectively, by dotted, dashed, and solid lines. arbitrary, in which case they should not appear in the final expression of $A_{n}$ given by Eq. (7), or they may be determined by the complementary condition.

For illustration, here are some concrete examples of the different classes. $\left[E_{1}^{S}, E_{2}^{S}, E_{3}^{S}\right]=[0,5,5]$ belongs to class $S_{1},[0,2,5]$ belongs to class $S_{3}^{\prime}$, and $[0,6,0]$ belongs to class $T_{2}$. The diagram Fig. 7 as a whole has $\left[E_{1}^{S}, E_{2}^{S}, E_{3}^{S}\right]=[4,4,2]$, so it belongs to class $T_{0}$.

If $\left[E_{1}^{S}, E_{2}^{S}, E_{3}^{S}\right]$ contains one even and two odd numbers, with the even number having color $a$, then $S$ belongs to class $S_{a}$. If they contain one odd and two even numbers, with the odd number having color $a$, then $S$ belongs to class $S_{a}^{\prime}$. In both cases, the propagator must have color $a$ to satisfy the topological requirement, so

$$
\begin{aligned}
& m_{S_{a}}^{2}=m_{S_{a}^{\prime}}^{2}=m_{a}^{2}, \quad m_{T_{0}}^{2}=M_{0}^{2}, \\
& m_{T_{a}}^{2}=M_{a}^{2} \quad(a=1,2,3) .
\end{aligned}
$$

\section{B. Complementary condition}

According to Eq. (42), the whole diagram must have $\left[E_{1}, E_{2}, E_{3}\right]$ all even or all odd, and $E_{a}+E_{b} \geq 2$ must be fulfilled for every pair of colors. These topological requirements can guarantee $m_{S}^{2}=m_{\bar{S}}^{2}$ only when all the masses in Eq. (43) are equal.

To see that, suppose $S=T_{0}$, then the topological requirement merely demands $\bar{S}$ to be either $T_{0}$, or $T_{a}$ if $E_{a}(\bar{S})$ is even. Thus $M_{0}^{2}=M_{a}^{2}$ for all $a$. If $S=S_{a}$, then $\bar{S}$ could be in class $S_{a}, S_{a}^{\prime}$, or class $T_{a}$ if $E_{a}(\bar{S})$ is odd. Hence $m_{a}^{2}=M_{a}^{2}$. Thus all the masses in Eq. (43) are equal.

\section{C. $\phi_{1} \phi_{2} \phi_{3}$ vs $\phi^{3}$}

With all the masses in Eq. (43) equal, the only difference between the $\phi_{1} \phi_{2} \phi_{3}$ and the $\phi^{3}$ amplitudes is that some Feynman diagrams allowed in $\phi^{3}$ would not be allowed in $\phi_{1} \phi_{2} \phi_{3}$. These are the diagrams violating the topological requirements discussed in Sec. VA.

\section{COMPOSITE THEORIES}

Once a set of $\mu_{i j}$ satisfying the complementary condition Eq. (10), the symmetry condition Eq. (18), and the covariant condition Eq. (19) is given, a Möbius invariant scalar amplitude can be computed from Eq. (7) and Eq. (8). Since these three conditions are all linear in $\mu_{i j}$, a linear combination of two such sets of $\mu_{i j}$ also satisfies the three conditions. In this way we can construct many composite theories from two or more known theories by making linear combinations. In this section we shall illustrate how to do that with a simple example. Clearly many other examples can be similarly constructed.

Let $\mu_{i j}=\alpha \mu_{i j}^{\prime}+\beta \mu_{i j}^{\prime \prime}$, where $\mu_{i j}^{\prime}$ comes from a Yukawa theory, with nucleon mass $m_{1}^{\prime}$ and pion mass $m_{0}^{\prime}$, and $\mu_{i j}^{\prime \prime}$ comes from another Yukawa theory, with nucleon mass $m_{1}^{\prime \prime}$ and pion mass $m_{0}^{\prime \prime}$. $\alpha$ and $\beta$ are arbitrary real parameters. 


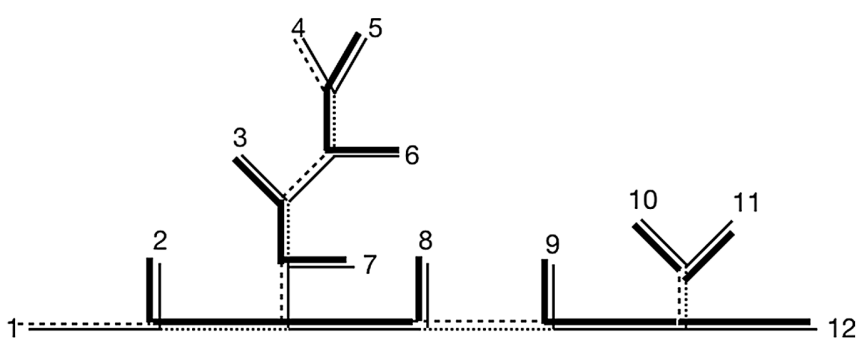

(a)

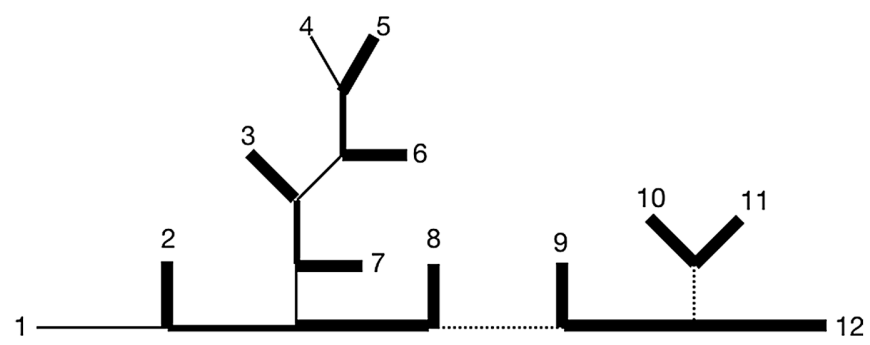

(b)

FIG. 8. A Feynman diagram of a double-Yukawa composite theory. (a) is drawn in terms of the two underlying theories, and (b) is drawn in terms of the composite particles. A dotted line in (b) is the new pion $\pi$, thin, medium, and thick solid lines represent, respectively, $N_{1}, N_{2}$, and $N^{*}$.

Let $\nu^{\prime}=0,1$ and $\nu^{\prime \prime}=0,1$ denote the nucleon number of the particles in the two basic theories. Then there are four kinds of particles in the composite theory: $\left(\nu^{\prime}, \nu^{\prime \prime}\right)=$ $(0,0),(1,0),(0,1),(1,1)$. We can interpret $\pi=(0,0)$ as a new (composite) pion with some mass (square) $m_{0}^{2}, N_{1}=$ $(1,0)$ as a new nucleon with mass $m_{1}^{2}, N_{2}=(0,1)$ as a second new nucleon with mass $m_{2}^{2}$, and $N^{*}=(1,1)$ as a new dinucleon resonance of mass $M^{2}$.

Figure 8(a) gives an example of a scattering diagram of the composite theory expressed in lines and vertices of the two basic theories. It is obtained by superimposing a diagram from the second basic theory on Fig. 5(a) of the first basic theory. As in Fig. 5(a), the nucleon and pion of the first theory are depicted by thin solid and dotted lines. The nucleon and pion of the second theory are depicted by thicker solid and dashed lines. Figure 8(b) is the same diagram expressed in terms of the composite particles $\pi, N_{1}, N_{2}$, and $N^{*}$, and the effective vertices $N_{1} N_{2} N^{*}$ and $N^{*} N^{*} \pi$. Dotted lines are composite pions $\pi$, and thin, medium, thick solid lines are the composite nucleons $N_{1}$, $N_{2}$ and the resonance $N^{*}$.

The propagator masses are

$$
\begin{aligned}
m_{S}^{2} & =\alpha m_{0}^{\prime 2}+\beta m_{0}^{\prime \prime 2}=m_{0}^{2}, & & \left(\nu^{\prime}, \nu^{\prime \prime}\right)=(\text { even, even }), \\
& =\alpha m_{0}^{\prime 2}+\beta m_{1}^{\prime \prime 2}=m_{2}^{2}, & & \left(\nu^{\prime}, \nu^{\prime \prime}\right)=(\text { even, odd }), \\
& =\alpha m_{1}^{\prime 2}+\beta m_{0}^{\prime \prime 2}=m_{1}^{2}, & & \left(\nu^{\prime}, \nu^{\prime \prime}\right)=(\text { odd }, \text { even }), \\
& =\alpha m_{1}^{\prime 2}+\beta m_{1}^{\prime \prime 2}=M^{2}, & & \left(\nu^{\prime}, \nu^{\prime \prime}\right)=(\text { odd }, \text { odd }) .
\end{aligned}
$$

TABLE I. Propagator masses $m_{S}^{2}$ obtained from Eq. (44) and Fig. 8(a) can be seen to be the same as those shown in Fig. 8(b).

\begin{tabular}{lccccc}
\hline \hline External lines & 12 & 45 & 456 & 3456 & 34567 \\
\hline$\left(\nu^{\prime}, \nu^{\prime \prime}\right)$ & $(2,1)$ & $(2,1)$ & $(3,2)$ & $(4,3)$ & $(5,4)$ \\
$m_{S}^{2}$ & $m_{2}^{2}$ & $m_{2}^{2}$ & $m_{1}^{2}$ & $m_{2}^{2}$ & $m_{1}^{2}$ \\
\hline \hline
\end{tabular}

\begin{tabular}{lllll}
\hline External lines & 1234567 & $(10)(11)$ & $(10)(11)(12)$ & $9(10)(11)(12)$
\end{tabular}

\begin{tabular}{lllll}
\hline$\left(\nu^{\prime}, \nu^{\prime \prime}\right)$ & $(7,5)$ & $(2,2)$ & $(3,3)$ & $(4,4)$
\end{tabular}

\begin{tabular}{lllll}
$m_{S}^{2}$ & $M^{2}$ & $m_{0}^{2}$ & $M^{2}$ & $m_{0}^{2}$ \\
\hline \hline
\end{tabular}

By adjusting the values of $\alpha, \beta, m_{0}^{\prime 2}, m_{1}^{\prime 2}, m_{0}^{\prime \prime 2}, m_{1}^{\prime \prime 2}$, we can have any value for the compound masses $m_{0}^{2}, m_{1}^{2}, m_{2}^{2}$, and $M^{2}$.

As a direct check, Table I shows the nine propagator masses in Fig. 8(b), obtained from this formula using the total $\left(\nu^{\prime}, \nu^{\prime \prime}\right)$ values read off from all the corresponding external lines in Fig. 8(a). They agree with what Fig. 8(b) shows.

\section{SCALAR QED}

The scattering function $f_{i}$ for scalar QED is identical to that of a Yukawa theory in Sec. III, provided we interpret $m_{1} \equiv m$ as the mass of the charged particle and $m_{0}=0$ as the mass of the photon. However, Feynman amplitudes in QED also contain nontrivial numerators describing photon polarizations as well as its derivative coupling to the charged particles. To implement that, the $\mathcal{I}_{n}$ factor in Eq. (7) must be different from Eq. (8). For an amplitude with two charged legs and $n-2$ photon legs, we propose to use

$$
\begin{aligned}
& \mathcal{I}_{n}=\frac{N}{\sigma_{(12 \cdots n)}}, \quad \text { where } \\
& N=\operatorname{Pf}^{\prime}(\Psi)=\frac{(-1)^{i+j}}{\sigma_{i j}} \operatorname{Pf}\left(\Psi_{i j}^{i j}\right) .
\end{aligned}
$$

$\Psi$ is the $2 n \times 2 n$ antisymmetric matrix

$$
\Psi=\left(\begin{array}{cccccccc}
0 & 0 & \cdots & 0 & -C_{11} & -C_{21} & \cdots & -C_{n 1} \\
0 & 0 & \cdots & 0 & -C_{12} & -C_{22} & \cdots & -C_{n 2} \\
\vdots & \vdots & & \vdots & \vdots & \vdots & & \vdots \\
0 & 0 & \cdots & 0 & -C_{1 n} & -C_{2 n} & \cdots & -C_{n n} \\
C_{11} & C_{12} & \cdots & C_{1 n} & 0 & 0 & \cdots & -1 / \sigma_{n 1} \\
C_{21} & C_{22} & \cdots & C_{2 n} & 0 & 0 & \cdots & 0 \\
\vdots & \vdots & & \vdots & \vdots & \vdots & & \vdots \\
C_{n 1} & C_{n 2} & \cdots & C_{n n} & 1 / \sigma_{n 1} & 0 & \cdots & 0
\end{array}\right),
$$


and $\Psi_{i j}^{i j}$ is $\Psi$ with the ith and $j$ th columns and rows removed. The matrix elements are

$$
\begin{aligned}
C_{i j} & =\frac{\epsilon_{i} \cdot k_{i}}{\sigma_{i j}}:=\frac{c_{i j}}{\sigma_{i j}}(i \neq j), \\
C_{i i} & =-\sum_{j \neq i} C_{i j},
\end{aligned}
$$

where $\epsilon_{i}$ is the polarization vector of photon $i$. Particles 1 and $n$ are the charged scalars, and the others are photons.

The motivation of this proposal is explained in Appendix C. In the rest of this section, the proposal will be explicitly verified for Compton scattering $(n=4)$ and for the process with a single photon emission $(n=5)$, by comparing the result of Eq. (7) with the Feynman amplitude obtained from Feynman rules.

\section{A. Compton scattering}

\section{Feynman amplitude}

Up to normalization, the Feynman amplitude of Fig. 9(a) is

$A_{F}^{a}=\frac{\epsilon_{2} \cdot\left(-q-k_{1}\right) \epsilon_{3} \cdot\left(k_{4}-q\right)}{\left(k_{3}+k_{4}\right)^{2}-m^{2}}=-4 \frac{\epsilon_{2} \cdot k_{1} \epsilon_{3} \cdot k_{4}}{\left(k_{3}+k_{4}\right)^{2}-m^{2}}$.

To be gauge invariant, we must also add in the cross diagram shown in Fig. 9(b), which is the same as Fig. 9(a) with $k_{2}$ and $k_{3}$ as well as $\epsilon_{2}$ and $\epsilon_{3}$ interchanged.

\section{CHY amplitude}

From Eq. (46) and Eq. (47) for $n=4$, one gets

$N=-\frac{1}{\sigma_{13} \sigma_{41}}\left(\frac{c_{24} c_{32}}{\sigma_{24} \sigma_{32}}+\frac{c_{21} c_{34}}{\sigma_{21} \sigma_{34}}+\frac{c_{23} c_{34}}{\sigma_{23} \sigma_{34}}+\frac{c_{24} c_{34}}{\sigma_{24} \sigma_{34}}\right)$.

Substituting this into Eq. (45), and choosing $\sigma_{r, s, t}$ to be $\sigma_{1,2,3}$ in Eq. (7), the CHY amplitude can be computed following the procedure outlined in Sec. II E. Instead of poles from $1 / \sigma_{(\alpha)}$, we must now look for poles in $N$ in the integration variable $\sigma_{4}$. Since line 2 is not neighboring to line 4 , poles from $\sim 1 / \sigma_{42}$ do not count. Moreover, as discussed in Sec. III D, we must also exclude poles that can lead to a would-be propagator of two pions. When applied to Fig. 9(a), this means that we should also ignore poles in $N$ proportional to $1 / \sigma_{41}$, leaving behind only poles of the form $1 / \sigma_{43}$ to be considered. Thus the first term in Eq. (49) does not contribute. For the remaining three terms, the residue of $1 / \sigma_{43}$ is proportional to the propagator $1 /\left[\left(k_{4}+k_{3}\right)^{2}-m^{2}\right]$. Any remaining $\sigma_{4}$ after the integration should be replaced by $\sigma_{3}$ because the integration region comes from the vicinity of $\sigma_{43}=0$. Up to a possible

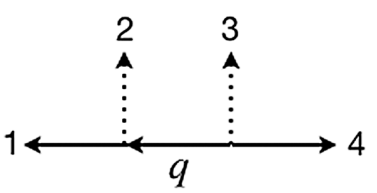

(a)

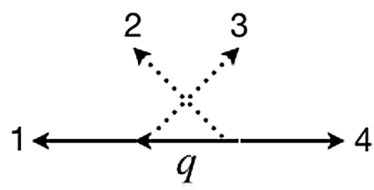

(b)
FIG. 9. Compton scattering diagrams. Solid and dotted lines are charged particles and photons, respectively.

normalization factor, the CHY amplitude from Eq. (7) is then

$$
\begin{aligned}
A_{\mathrm{CHY}}^{a}= & \frac{\sigma_{(123)}}{\left(k_{4}+k_{3}\right)^{2}-m^{2}}\left(\frac{1}{\sigma_{13} \sigma_{31}}\right) \\
& \times\left(\frac{c_{21} c_{34}}{\sigma_{21}}+\frac{c_{23} c_{34}}{\sigma_{23}}+\frac{c_{24} c_{34}}{\sigma_{23}}\right),
\end{aligned}
$$

in which the $\sigma_{(123)}=\sigma_{(r s t)}$ factor comes from the integrand of Eq. (7). Using the momentum conservation relation $c_{21}=-\left(c_{23}+c_{24}\right)$, we end up with

$$
A_{\mathrm{CHY}}^{a}=\frac{c_{21} c_{34}}{\left(k_{4}+k_{3}\right)^{2}-m^{2}},
$$

which agrees with the Feynman amplitude $A_{f}^{q}$ in Eq. (48) up to a normalization factor. The correctness of the prescription Eq. (45) for $n=4$ is thereby verified.

The cross diagram Fig. 9(b) can be obtained either by interchanging 2 and 3 in Eq. (50) or by changing $\mathcal{I}_{n}$ in Eq. (45) to $N / \sigma_{(1324)}$.

\section{B. Photon emission}

\section{Feynman amplitude}

Up to normalization, the Feynman amplitude of Fig. 10 is

$$
\begin{aligned}
& A_{F}=\frac{\epsilon_{2} \cdot\left(-q_{1}-k_{1}\right) \epsilon_{3} \cdot\left(q_{2}-q_{1}\right) \epsilon_{4} \cdot\left(k_{5}+q_{2}\right)}{\left[\left(k_{3}+k_{4}\right)^{2}-m^{2}\right]\left[\left(k_{4}+k_{5}+k_{5}\right)^{2}-m^{2}\right]} \\
& =-4 \frac{\epsilon_{2} \cdot k_{1} \epsilon_{3} \cdot\left(k_{4}+k_{5}-k_{1}-k_{2}\right) \epsilon_{4} \cdot k_{5}}{\left[\left(k_{4}+k_{5}\right)^{2}-m^{2}\right]\left[\left(k_{3}+k_{4}+k_{5}\right)^{2}-m^{2}\right]} \\
& =-8 \frac{c_{21}\left(c_{34}+c_{35}\right) c_{45}}{\left[\left(k_{4}+k_{5}\right)^{2}-m^{2}\right]\left[\left(k_{3}+k_{4}+k_{5}\right)^{2}-m^{2}\right]} \text {. }
\end{aligned}
$$

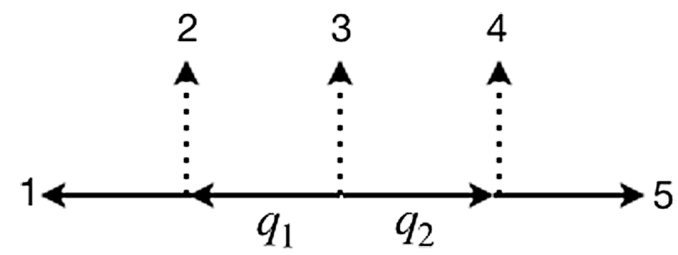

FIG. 10. A tree diagram for a single photon emission in photon proton scattering. Solid and dotted lines are charged particles and photons, respectively. 
Again for gauge invariance, we must add in five other cross diagrams obtained by permuting 2, 3, 4, but we will not consider them here.

\section{CHY amplitude}

From Eq. (45) and Eq. (46) for $n=5$, one gets

$$
\begin{aligned}
N= & -\frac{c_{25} c_{32} c_{41}}{\sigma_{13} \sigma_{25} \sigma_{32} \sigma_{41} \sigma_{51}}-\frac{c_{21} c_{35} c_{41}}{\sigma_{13} \sigma_{21} \sigma_{35} \sigma_{41} \sigma_{51}}-\frac{c_{23} c_{35} c_{41}}{\sigma_{13} \sigma_{23} \sigma_{35} \sigma_{41} \sigma_{51}}-\frac{c_{24} c_{35} c_{41}}{\sigma_{13} \sigma_{24} \sigma_{35} \sigma_{41} \sigma_{51}}-\frac{c_{25} c_{35} c_{41}}{\sigma_{13} \sigma_{25} \sigma_{35} \sigma_{41} \sigma_{51}} \\
& -\frac{c_{25} c_{32} c_{42}}{\sigma_{13} \sigma_{25} \sigma_{32} \sigma_{42} \sigma_{51}}-\frac{c_{25} c_{34} c_{42}}{\sigma_{13} \sigma_{25} \sigma_{34} \sigma_{42} \sigma_{51}}-\frac{c_{21} c_{35} c_{42}}{\sigma_{13} \sigma_{21} \sigma_{35} \sigma_{42} \sigma_{51}}-\frac{c_{23} c_{35} c_{42}}{\sigma_{13} \sigma_{23} \sigma_{35} \sigma_{42} \sigma_{51}}-\frac{c_{25} c_{35} c_{42}}{\sigma_{13} \sigma_{25} \sigma_{35} \sigma_{42} \sigma_{51}} \\
& -\frac{c_{25} c_{32} c_{43}}{\sigma_{13} \sigma_{25} \sigma_{32} \sigma_{43} \sigma_{51}}-\frac{c_{21} c_{35} c_{43}}{\sigma_{13} \sigma_{21} \sigma_{35} \sigma_{43} \sigma_{51}}-\frac{c_{23} c_{35} c_{43}}{\sigma_{13} \sigma_{23} \sigma_{35} \sigma_{43} \sigma_{51}}-\frac{c_{24} c_{35} c_{43}}{\sigma_{13} \sigma_{24} \sigma_{35} \sigma_{43} \sigma_{51}}-\frac{c_{25} c_{35} c_{43}}{\sigma_{13} \sigma_{25} \sigma_{35} \sigma_{43} \sigma_{51}} \\
& -\frac{c_{24} c_{32} c_{45}}{\sigma_{13} \sigma_{24} \sigma_{32} \sigma_{45} \sigma_{51}}-\frac{c_{25} c_{32} c_{45}}{\sigma_{13} \sigma_{25} \sigma_{32} \sigma_{45} \sigma_{51}}-\frac{c_{21} c_{34} c_{45}}{\sigma_{13} \sigma_{21} \sigma_{34} \sigma_{45} \sigma_{51}}-\frac{c_{23} c_{34} c_{45}}{\sigma_{13} \sigma_{23} \sigma_{34} \sigma_{45} \sigma_{51}}-\frac{c_{24} c_{34} c_{45}}{\sigma_{13} \sigma_{24} \sigma_{34} \sigma_{45} \sigma_{51}} \\
& -\frac{c_{25} c_{34} c_{45}}{\sigma_{13} \sigma_{25} \sigma_{34} \sigma_{45} \sigma_{51}}-\frac{c_{21} c_{35} c_{45}}{\sigma_{13} \sigma_{21} \sigma_{35} \sigma_{45} \sigma_{51}}-\frac{c_{23} c_{35} c_{45}}{\sigma_{13} \sigma_{23} \sigma_{35} \sigma_{45} \sigma_{51}}-\frac{c_{24} c_{35} c_{45}}{\sigma_{13} \sigma_{24} \sigma_{35} \sigma_{45} \sigma_{51}}-\frac{c_{25} c_{35} c_{45}}{\sigma_{13} \sigma_{25} \sigma_{35} \sigma_{45} \sigma_{51}} .
\end{aligned}
$$

As before, choose $\sigma_{r, s, t}=\sigma_{1,2,3}$ in Eq. (7), and then search for poles in $N$ in the integration variables $\sigma_{4}$ and $\sigma_{5}$. Poles with non-neighboring lines do not count, and poles leading up to pure pion propagators should also be ignored. Therefore the relevant poles in $N$ only come from terms containing a $1 /\left(\sigma_{35} \sigma_{45}\right)$ factor or a $1 /\left(\sigma_{34} \sigma_{45}\right)$ factor, both giving rise to a residue proportional to $1 /\left[\left(k_{4}+k_{5}\right)^{2}-m^{2}\right] \times$ $\left[\left(k_{3}+k_{4}+k_{5}\right)^{2}-m^{2}\right]$. All left over $\sigma_{4}$ and $\sigma_{5}$ factors after the integration should be set equal to $\sigma_{3}$.

With that in mind, of the 25 terms in $N$, only terms 18 to 25 contribute. Up to a possible normalization factor, the CHY amplitude for $n=5$ is then

$$
\begin{aligned}
A_{C H Y}= & \frac{\sigma_{(123)} B c_{45}}{\left[\left(k_{4}+k_{5}\right)^{2}-m^{2}\right]\left[\left(k_{3}+k_{4}+k_{5}\right)^{2}-m^{2}\right]}, \\
B= & -\frac{1}{\sigma_{13} \sigma_{31}}\left(\frac{c_{21} c_{34}}{\sigma_{21}}+\frac{c_{23} c_{34}}{\sigma_{23}}+\frac{c_{24} c_{34}}{\sigma_{23}}+\frac{c_{25} c_{34}}{\sigma_{23}}\right) \\
& -\frac{1}{\sigma_{13} \sigma_{31}}\left(\frac{c_{21} c_{35}}{\sigma_{21}}+\frac{c_{23} c_{35}}{\sigma_{23}}+\frac{c_{24} c_{35}}{\sigma_{23}}+\frac{c_{25} c_{35}}{\sigma_{23}}\right) \\
= & -c_{21}\left(c_{34}+c_{35}\right) \frac{1}{\sigma_{13} \sigma_{31}}\left(\frac{1}{\sigma_{21}}-\frac{1}{\sigma_{23}}\right) \\
= & \frac{c_{21}\left(c_{34}+c_{35}\right)}{\sigma_{13} \sigma_{21} \sigma_{23}},
\end{aligned}
$$

in which momentum conservation $c_{21}=-\left(c_{23}+c_{24}+\right.$ $c_{25}$ ) has been used. Substituting $B$ into $A_{\mathrm{CHY}}$, one gets

$$
A_{\mathrm{CHY}}=\frac{c_{21}\left(c_{34}+c_{35}\right) c_{45}}{\left[\left(k_{4}+k_{5}\right)^{2}-m^{2}\right]\left[\left(k_{3}+k_{4}+k_{5}\right)^{2}-m^{2}\right]},
$$

which up to a normalization factor is the same as the Feynman amplitude $A_{F}$ in Eq. (51), thereby verifying the correctness of Eq. (45) for $n=5$.

\section{DISK AND SPHERE INTEGRALS}

A disk integral

$$
\begin{aligned}
Z_{P}\left(q_{1} q_{2} \cdots q_{n}\right)= & \left(\alpha^{\prime}\right)^{n-3} \int_{D(P)} \frac{d z_{1} d z_{2} \cdots d z_{n}}{\operatorname{vol}(S L(2, R))} \\
& \times \frac{\prod_{i<j}\left|z_{i j}\right|^{\alpha^{\prime} k_{i} \cdot k_{j}}}{z_{q_{1} q_{2}} z_{q_{2} q_{3}} \cdots z_{q_{n-1} q_{n}} z_{q_{n} q_{1}}}
\end{aligned}
$$

was introduced in $[75,76]$ to connect a Yang-Mills field theory amplitude with an open string amplitude. Following [60], the integration variables $\sigma_{i}$ are now denoted as $z_{i}$. $\alpha^{\prime}$ is the Regge slope, and $k_{i}$ are the lightlike external momenta. The integration domain is the real line with the integration variables ordered according to a permutation $P=\left\{p_{1} p_{2} \cdots p_{n}\right\} \in S_{n}$,

$$
\begin{aligned}
D(P)= & \left\{\left(z_{1} z_{2} \cdots z_{n}\right) \in R^{n} \mid-\infty<z_{p_{1}}<z_{p_{2}}<\cdots\right. \\
& \left.<z_{p_{n}}<\infty\right\} .
\end{aligned}
$$

The $Z$ function depends on the permutation $P$, and also on another permutation $Q=\left\{q_{1} q_{2} \cdots q_{n}\right\} \in S_{n}$. The integrand is invariant under a $\operatorname{SL}(2, \mathrm{R})$ transformation, which is the Möbius transformation with real coefficients.

The $Z$ function possesses the following properties [73]:

$$
\begin{gathered}
Z_{P}\left(q_{1} q_{2} \cdots q_{n}\right)=Z_{P}\left(q_{2} q_{3} \cdots q_{1}\right), \\
Z_{P}\left(q_{1} q_{2} \cdots q_{n}\right)=(-)^{n} Z_{P}\left(q_{n} q_{n-1} \cdots q_{1}\right), \\
Z_{P}(1, A, n, B)=(-)^{|B|} \sum_{A \amalg \tilde{B}} Z_{P}(1, \sigma, n), \quad \forall A, B, \\
0=\sum_{k=2}^{n-1} k_{q_{1}} \cdot\left(k_{q_{2}}+k_{q_{3}}+\cdots+k_{q_{j}}\right) \\
\times Z_{P}\left(q_{2} q_{3} \cdots q_{j} q_{1} q_{j+1} \cdots q_{n}\right),
\end{gathered}
$$




$$
Z_{p_{1} p_{2} \cdots p_{n}}(Q)=Z_{p_{2} p_{3} \cdots p_{n} p_{1}}(Q)=(-1)^{n} Z_{p_{n} \cdots p_{2} p_{1}}(Q),
$$

$$
\begin{aligned}
0= & \sum_{j=1}^{n-1} \exp \left[i \pi \alpha^{\prime} k_{p_{1}} \cdot\left(k_{p_{2}}+k_{p_{3}}+\cdots+k_{p_{j}}\right)\right] \\
& \times Z_{p_{2} p_{3} \cdots p_{j} p_{1} p_{j+1} \cdots p_{n}},
\end{aligned}
$$

where $\tilde{B}$ is the transpose of the set $B$, and $A \sqcup \tilde{B}$ is the shuffle product between set $A$ and set $\tilde{B}$. In the zero Regge slope limit, $Z_{P}(Q)$ is essentially the scalar amplitude Eq. (7), with $P=\{12 \cdots n\}$ and $Q=\alpha$.

The $Z$ function can be generalized to massive and offshell situations by replacing $k_{i} \cdot k_{j}$ in Eq. (53) with $a_{i j}$ of the previous sections. Since $a_{i i}=0$ and $\sum_{j \neq i} a_{i j}=0$, the Koba-Nielsen factor $\left|z_{i j}\right|^{\alpha^{\prime} a_{i j}}$ remains invariant under a Möbius transformation, thereby leaving the integral in Eq. (53) well defined. This modified $Z$ function still possesses all the same properties, Eq. (54) to Eq. (59), except that the momentum dot products in Eq. (57) and Eq. (59) should be replaced by the sum of $a_{i j}$ factors:

$$
\begin{aligned}
& k_{q_{1}} \cdot\left(k_{q_{2}}+k_{q_{3}}+\cdots+k_{q_{j}}\right) \rightarrow \sum_{j=2}^{k} a_{q_{1} q_{j}}, \\
& k_{p_{1}} \cdot\left(k_{p_{2}}+k_{p_{3}}+\cdots+k_{p_{j}}\right) \rightarrow \sum_{j=2}^{k} a_{p_{1} p_{j}} .
\end{aligned}
$$

In the zero Regge slope limit, it again reduces to the field theory amplitude defined by the same $a_{i j}$.

With these similarities, it would be interesting to find out whether modified string amplitudes and/or new effective field theories can be sensibly constructed with the help of the modified $Z$ function.

A similar remark applies to the modified sphere function, defined by

$$
\begin{aligned}
& J_{P}\left(q_{1} q_{2} \cdots q_{n}\right) \\
& =\left(\alpha^{\prime}\right)^{n-3} \int_{C^{n}} \frac{d^{2} z_{1} d^{2} z_{2} \cdots d^{2} z_{n}}{\operatorname{vol}(S L(2, C))} . \\
& \quad \times \frac{\prod_{i<j}\left|z_{i j}\right| 2 \alpha^{\prime} a_{i j}}{\left(z_{p_{1} p_{2}} z_{p_{2} p_{3}} \cdots z_{p_{n-1} p_{n}} z_{p_{n} p_{1}}\right)\left(z_{q_{1} q_{2}} z_{q_{2} q_{3}} \cdots z_{q_{n-1} q_{n}} z_{q_{n} q_{1}}\right)} .
\end{aligned}
$$

When the corresponding field theory is massless and onshell, $a_{i j}=k_{i} \cdot k_{j}$, the $J$ function returns to the original one in [74].

\section{SUMMARY}

By modifying the scattering equations used in the CHY tree amplitudes, we extended the formalism to many off-shell scalar amplitudes carrying nonzero masses. The modification consists of changing $k_{i} \cdot k_{j}$ to an arbitrary $a_{i j}=k_{i} \cdot k_{j}+\rho_{i j}+\mu_{i j}$, with $\sum_{j \neq i} a_{i j}=0$. The term $\rho_{i j}$ provides an off-shell extension, and the term $\mu_{i j}$ specifies how nonzero masses enter cubic interactions. The same modification can be applied to the Koba-Nielsen factor of disk and sphere functions, which may be useful in generalizing string related amplitudes. Future work includes how amplitudes involving higher spins can be similarly constructed, and how an off-shell extension can be used to study amplitudes with any number of loops.

\section{APPENDIX A: MASSES AND OFF-SHELL MOMENTA COMING FROM EXTRA DIMENSIONS}

Consider an $n$-particle scattering amplitude with external momenta $\hat{k}_{i}$ in a higher dimensional space, and suppose $\hat{k}_{i}^{2}=0$ for all $i$. Decompose $\hat{k}_{i}=\left(k_{i}, k_{i}^{\prime}\right)$ into a fourdimensional component $k_{i}$ and an extradimensional component $k_{i}^{\prime}$. Since $k_{i}^{2}=-k_{i}^{\prime 2}$ may take on any value, a nonzero mass $m$ and/or an off-shell four-momentum can be produced in real spacetime. We show in this Appendix that masses produced this way do not always yield the right propagators in a massive theory, though it might do so if only certain particles in the amplitude are massive. Similar arguments apply also to off-shell momenta.

Consider a theory whose particles carry a mass $m$. Label the external lines cyclically in the natural order $i=1$, $2, \ldots, n$. For any $p$ and any $q \leq n-2$, there is always an inverse propagator $\left(\sum_{i=p}^{p+q-1} k_{i}\right)^{2}-m^{2}$ present in some tree diagram. If masses come strictly from the extradimensional momenta $\hat{k}_{i}$, then such an inverse propagator must be $\left(\sum_{i=p}^{p+q-1} \hat{k}_{i}\right)^{2}=\left(\sum_{i=p}^{p+q-1} k_{i}\right)^{2}+\left(\sum_{i=p}^{p+q-1} k_{i}^{\prime}\right)^{2}$. This requires

$$
\left(\sum_{i=p}^{p+q-1} k_{i}^{\prime}\right)^{2}=-m^{2}
$$

for any $p$ and any $q \leq n-2$. This turns out to be impossible for a fixed extra dimension and a large enough $n$.

Using Eq. (A1) and $k_{p}^{\prime 2}=-m^{2}$, we get

$$
2 k_{p}^{\prime} \cdot\left(\sum_{i=p+1}^{p+q-1} k_{i}^{\prime}\right)=m^{2}
$$

for any $2 \leq q \leq n-2$. Hence $k_{p}^{\prime} \cdot k_{i}^{\prime}=0$ for all $i \geq p+2$ as long as $i-p+1 \leq n-2$. This cannot happen if $n$ is large enough, because $k_{p}^{\prime} \cdot k_{i}^{\prime}=0$ can occur only for $(d-1) i$ 's in a $d$-dimensional extra dimension.

This concludes the proof that we cannot obtain all propagators of all Feynman diagrams correctly if masses 
for a massive field theory come solely from the extra dimensions.

\section{APPENDIX B: COVARIANCE AND SUM RULES}

If $a_{i j}$ satisfies the constraints $a_{i i}=0$ and $\sum_{j} a_{i j}=0$, then $\hat{f}_{i}(\sigma)$ will transform covariantly as in Eq. (4), and the sum rules of Eq. (6) will be satisfied.

To prove these assertions, note that under a Möbius transformation given by Eq. (3), $1 /\left(\sigma-\sigma_{i}\right) \rightarrow \lambda \lambda_{i} /\left(\sigma-\sigma_{i}\right)$ where $\lambda=(\gamma \sigma+\delta)$ and $\lambda_{i}=\left(\gamma \sigma_{i}+\delta\right)$. Hence

$$
\begin{aligned}
\hat{f}_{i}(\sigma) & =\sum_{j=1, j \neq i} \frac{a_{i j}}{\sigma-\sigma_{j}} \rightarrow \lambda \sum_{j \neq i} \frac{a_{i j} \lambda_{j}}{\sigma-\sigma_{j}} \\
& =\lambda \sum_{j \neq i} \frac{a_{i j}}{\sigma-\sigma_{j}}\left[\lambda-\gamma\left(\sigma-\sigma_{j}\right)\right]=\lambda^{2} \hat{f}_{i}(\sigma)
\end{aligned}
$$

because $\sum_{j} a_{i j}=0$.

For the sum rules, recall that $f_{i}=\hat{f}_{i}\left(\sigma_{i}\right)=$ $\sum_{j \neq i} a_{i j} /\left(\sigma_{i}-\sigma_{j}\right)$; hence

$$
\begin{aligned}
\sum_{i} f_{i}= & \sum_{i \neq j} \frac{a_{i j}}{\sigma_{i}-\sigma_{j}}=0, \\
\sum_{i} f_{i} \sigma_{i}= & \sum_{i \neq j} \frac{a_{i j} \sigma_{i}}{\sigma_{i}-\sigma_{j}}=\frac{1}{2} \sum_{i \neq j} \frac{a_{i j}\left(\sigma_{i}-\sigma_{j}\right)}{\sigma_{i}-\sigma_{j}}=0, \\
\sum_{i} f_{i} \sigma_{i}^{2}= & \sum_{i \neq j} \frac{a_{i j} \sigma_{i}^{2}}{\sigma_{i}-\sigma_{j}}=\sum_{i \neq j} \frac{a_{i j} \sigma_{i}\left(\sigma_{i}-\sigma_{j}\right)}{\sigma_{i}-\sigma_{j}} \\
& +\sum_{i \neq j} \frac{a_{i j} \sigma_{i} \sigma_{j}}{\sigma_{i}-\sigma_{j}}=0 .
\end{aligned}
$$

The last of the first equation is 0 because the summand is antisymmetric in $i, j$. The last term of the second equation is zero because $\sum_{j} a_{i j}=0$. The first term of the last expression of the last equation is zero because $\sum_{j} a_{i j}=0$, and the second term of that expression is zero because the summand is antisymmetric in $i$ and $j$.

\section{APPENDIX C: NUMERATOR FACTOR FOR QED}

The numerator factor $N$ in Eq. (45) comes from a comparison between the Feynman rules for QED and those for gluon scattering.

For gluon scatterings, there is the four-gluon vertex in Fig. 11(e), and the three-gluon vertex Fig. 11(a), containing three terms shown separately in diagrams (b), (c), and (e). The vertex factor for Fig. 11(c) is identical to the vertex factor in scalar QED, shown in Fig. 11(f), except for $\epsilon_{1} \cdot \epsilon_{3}$.

A gluon amplitude is a function of $\epsilon_{i} \cdot \epsilon_{j}, \epsilon_{i} \cdot k_{j}$, and $k_{i} \cdot k_{j}$. In the CHY theory [2,3], the numerator of the amplitude comes from the reduced Pfaffian of a $2 n \times 2 n$ antisymmetric matrix of the form

$$
\Psi=\left(\begin{array}{cc}
A & -C^{T} \\
C & B
\end{array}\right)
$$

where for $i \neq j, A_{i j}=k_{i} \cdot k_{j} / \sigma_{i j}, C_{i j}=\epsilon_{i} \cdot k_{j} / \sigma_{i j}$, and $B_{i j}=\epsilon_{i} \cdot \epsilon_{j} / \sigma_{i j}$. A scalar QED amplitude with two charged scalars occupying lines 1 and $n$, such as Fig. 9 or Fig. 10, contains $\epsilon_{i} \cdot k_{j}$ factors but no $\epsilon_{i} \cdot \epsilon_{j}$ nor $k_{i} \cdot k_{j}$ factors in the numerator. Since Fig. 11(c) is essentially the same as Fig. 11(f), we may obtain the numerator factor $N$ in such QED diagrams from Eq. (C1) by putting $A=0$, and $B=0$ except for the $\epsilon_{1} \cdot \epsilon_{n} / \sigma_{1 n}$ and the $\epsilon_{n} \cdot \epsilon_{1} / \sigma_{n 1}$ elements. This is then the $N$ factor in Eq. (45).

There is, however, a caveat in using $N$. A number of gluons can merge to form a gluon propagator, but photons in a tree diagram cannot merge to form photon propagators. Hence those poles in $N$ that lead to the phantom photon propagators must be excluded by hand. Other than this, $N$ has another imperfection. The reduced Pfaffian for the gluon matrix in Eq. (C1) is gauge invariant, but this is not so for the photon matrix in Eq. (46).

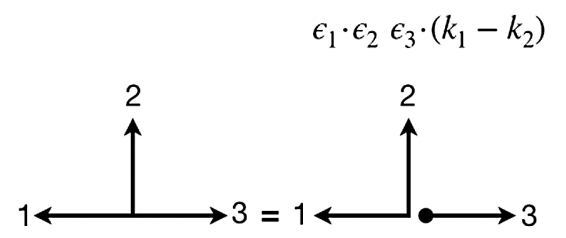

(a)

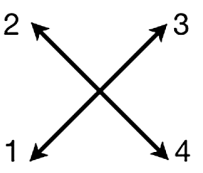

(e) (b)

(c)

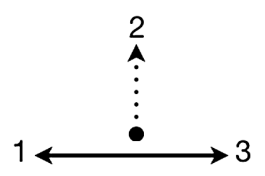

(f)

FIG. 11. Vertex factors for QCD shown in (a) and (e), and for scalar QED shown in (f). 
Moreover, this argument to produce $N$ no longer works when two pairs of charged legs are present, for two different reasons. First, there would now be two $\epsilon_{i} \cdot \epsilon_{j}$ factors in the $B$ block for the two charged pairs. If the factor $B$ in Eq. (C1) contains two such pairs of $\epsilon_{i} \cdot \epsilon_{j}$, then their product would appear somewhere in the reduced Pfaffian. In the case of gluon scattering, this product could come from the four-gluon vertices Fig. 11(e), or terms such as Fig. 11(b) or Fig. 11(d). In the case of scalar QED, all these diagrams are absent, so this term would be extraneous and wrong. The second reason why that does not work is because $k_{i} \cdot k_{j}$ factors would appear when the two pairs exchange a photon, so the $A$ block in Eq. (C1)can no longer be dropped. However, Möbius invariance demands $A$ to be $a_{i j} / \sigma_{i j}$ when masses are present [70], not just $k_{i} \cdot k_{j} / \sigma_{i j}$. Then mass factors $\mu_{i j} / \sigma_{i j} \sim m / \sigma_{i j}$ would also appear in $A$ and in the numerator of the scattering amplitude. That is wrong and should not happen. For both of these reasons, the $N$ factors for scalar QED can no longer be obtained so simply from the gluon $N$ factor when more than one pair of charged particles are present.
[1] F. Cachazo, S. He, and E. Y. Yuan, Scattering equations and KLT orthogonality, Phys. Rev. D 90, 065001 (2014).

[2] F. Cachazo, S. He, and E. Y. Yuan, Scattering of Massless Particles in Arbitrary Dimensions, Phys. Rev. Lett. 113, 171601 (2014).

[3] F. Cachazo, S. He, and E. Y. Yuan, Scattering of massless particles: Scalars, gluons and gravitons, J. High Energy Phys. 07 (2014) 033.

[4] F. Cachazo, S. He, and E. Y. Yuan, Einstein-Yang-Mills scattering amplitudes from scattering equations, J. High Energy Phys. 01 (2015) 121.

[5] F. Cachazo, S. He, and E. Y. Yuan, Scattering equations and matrices: From Einstein to Yang-Mills, DBI and NLSM, J. High Energy Phys. 07 (2015) 149.

[6] L. Dolan and P. Goddard, Proof of the formula of Cachazo, $\mathrm{He}$ and Yuan for Yang-Mills tree amplitudes in arbitrary dimension, J. High Energy Phys. 01 (2014) 152; 05 (2014) 010 .

[7] F. Cachazo and H. Gomez, Computation of contour integrals on $\mathcal{M}_{0, n}$, J. High Energy Phys. 04 (2016) 108.

[8] C. Baadsgaard, N. E. J. Bjerrum-Bohr, J. L. Bourjaily, and P. H. Damgaard, Integration rules for scattering equations, J. High Energy Phys. 09 (2015) 129.

[9] C. Baadsgaard, N. E. J. Bjerrum-Bohr, J. L. Bourjaily, and P. H. Damgaard, Scattering equations and Feynman diagrams, J. High Energy Phys. 09 (2015) 136.

[10] C. Baadsgaard, N. E. J. Bjerrum-Bohr, J. L. Bourjaily, P. H. Damgaard, and B. Feng, Integration rules for loop scattering equations, J. High Energy Phys. 11 (2015) 080.

[11] C.S. Lam and Y. P. Yao, The role of Mbius constants and scattering functions in CHY scalar amplitudes, Phys. Rev. D 93, 105004 (2016).

[12] C.S. Lam and Y.-P. Yao, Evaluation of the CHY gauge amplitude, Phys. Rev. D 93, 105008 (2016).

[13] R. Huang, B. Feng, M.-x. Luo, and C.-J. Zhu, Feynman rules of higher-order poles in CHY construction, J. High Energy Phys. 06 (2016) 013.

[14] N. E. J. Bjerrum-Bohr, J. L. Bourjaily, P. H. Damgaard, and B. Feng, Analytic representations of Yang-Mills amplitudes, Nucl. Phys. 10, 012 (2016); Nucl. Phys. B913, 964 (2016).
[15] S. He and Y. Zhang, New formulas for amplitudes from higher-dimensional operators, J. High Energy Phys. 02 (2017) 019.

[16] G. Chen, Y. K. E. Cheung, T. Wang, and F. Xu, A combinatoric shortcut to evaluate $\mathrm{CHY}$-forms, J. High Energy Phys. 06 (2017) 015.

[17] R. Huang, Y.-J. Du, and B. Feng, Understanding the cancelation of double poles in the Pfaffian of $\mathrm{CHY}$ formalism, J. High Energy Phys. 06 (2017) 133.

[18] X. Gao, S. He, and Y. Zhang, Labelled tree graphs, Feynman diagrams and disk integrals, J. High Energy Phys. 11 (2017) 144.

[19] C. S. Lam, Pfaffian diagrams for gluon tree amplitudes, Phys. Rev. D 98, 076002 (2018).

[20] C. Kalousios, Massless scattering at special kinematics as Jacobi polynomials, J. Phys. A 47, 215402 (2014).

[21] S. Weinzierl, On the solutions of the scattering equations, J. High Energy Phys. 04 (2014) 092.

[22] L. Dolan and P. Goddard, The polynomial form of the scattering equations, J. High Energy Phys. 07 (2014) 029.

[23] Y.-H. He, C. Matti, and C. Sun, The scattering variety, J. High Energy Phys. 10 (2014) 135.

[24] S. G. Naculich, Scattering equations and virtuous kinematic numerators and dual-trace functions, J. High Energy Phys. 07 (2014) 143.

[25] C. S. Lam, Permutation symmetry of the scattering equations, Phys. Rev. D 91, 045019 (2015).

[26] R. Huang, J. Rao, B. Feng, and Y.-H. He, An algebraic approach to the scattering equations, J. High Energy Phys. 12 (2015) 056.

[27] M. Sogaard and Y. Zhang, An algebraic approach to the scattering equations, Phys. Rev. D 93, 105009 (2016).

[28] C. Cardona and C. Kalousios, An algebraic approach to the scattering equations, J. High Energy Phys. 01 (2016) 178.

[29] C. Cardona and C. Kalousios, Elimination and recursions in the scattering equations, Phys. Lett. 03B, 003 (2016); Phys. Lett. B 756, 180 (2016).

[30] L. Dolan and P. Goddard, General solution of the scattering equations, J. High Energy Phys. 10 (2016) 149.

[31] Y.-j. Du, F. Teng, and Y.-s. Wu, CHY formula and MHV amplitudes, J. High Energy Phys. 05 (2016) 086. 
[32] M. Zlotnikov, Polynomial reduction and evaluation of treeand loop-level CHY amplitudes, J. High Energy Phys. 08 (2016) 143.

[33] F. Cachazo, S. Mizera, and G. Zhang, Scattering equations: Real solutions and particles on a line, J. High Energy Phys. 03 (2017) 151.

[34] S. Mizera, Scattering Amplitudes from Intersection Theory, Phys. Rev. Lett. 120, 141602 (2018).

[35] B. U. W. Schwab and A. Volovich, Subleading Soft Theorem in Arbitrary Dimension from Scattering Equations, Phys. Rev. Lett. 113, 101601 (2014).

[36] F. Cachazo, S. He, and E. Y. Yuan, New double soft emission theorems, Phys. Rev. D 92, 065030 (2015).

[37] F. Cachazo, P. Cha, and S. Mizera, Extensions of theories from soft limits, J. High Energy Phys. 06 (2016) 170.

[38] A. P. Saha, Double soft theorem for perturbative gravity, J. High Energy Phys. 09 (2016) 165.

[39] D. Nandan, J. Plefka, and W. Wormsbecher, Collinear limits beyond the leading order from the scattering equations, J. High Energy Phys. 02 (2017) 038.

[40] S. He and E. Y. Yuan, One-loop scattering equations and amplitudes from forward limit, Phys. Rev. D 92, 105004 (2015).

[41] C. Baadsgaard, N. E. J. Bjerrum-Bohr, J. L. Bourjaily, S. Caron-Huot, P. H. Damgaard, and B. Feng, New Representations of the Perturbative S-Matrix, Phys. Rev. Lett. 116, 061601 (2016).

[42] Y. Geyer, L. Mason, R. Monteiro, and P. Tourkine, One-loop amplitudes on the Riemann sphere, J. High Energy Phys. 03 (2016) 114.

[43] F. Cachazo, S. He, and E. Y. Yuan, One-loop corrections from higher dimensional tree amplitudes, J. High Energy Phys. 08 (2016) 008.

[44] B. Feng, CHY-construction of planar loop integrands of cubic scalar theory, J. High Energy Phys. 05 (2016) 061.

[45] Y. Geyer, L. Mason, R. Monteiro, and P. Tourkine, Twoloop scattering amplitudes from the Riemann sphere, Phys. Rev. D 94, 125029 (2016).

[46] H. Gomez, Quadratic Feynman loop integrands from massless scattering equations, Phys. Rev. D 95, 106006 (2017).

[47] H. Gomez, C. Lopez-Arcos, and P. Talavera, One-loop ParkeTaylor factors for quadratic propagators from massless scattering equations, J. High Energy Phys. 10 (2017) 175.

[48] L. Mason and D. Skinner, Ambitwistor strings and the scattering equations, J. High Energy Phys. 07 (2014) 048.

[49] Y. Geyer, A. E. Lipstein, and L. J. Mason, Ambitwistor Strings in 4-Dimensions, Phys. Rev. Lett. 113, 081602 (2014).

[50] H. Gomez, $\Lambda$ scattering equations, J. High Energy Phys. 06 (2016) 101.

[51] C. Cheung, C.-H. Shen, and C. Wen, Unifying relations for scattering amplitudes, J. High Energy Phys. 02 (2018) 095.

[52] L. de la Cruz, A. Kniss, and S. Weinzierl, Properties of scattering forms and their relation to associahedra, J. High Energy Phys. 03 (2018) 064.

[53] N. Arkani-Hamed, Y. Bai, S. He, and G. Yan, Scattering forms and the positive geometry of kinematics, color and the worldsheet, J. High Energy Phys. 05 (2018) 096.
[54] S. He, G. Yan, C. Zhang, and Y. Zhang, Scattering forms, worldsheet forms and amplitudes from subspaces, J. High Energy Phys. 08 (2018) 040.

[55] S. He and C. Zhang, Notes on scattering amplitudes as differential forms, J. High Energy Phys. 10 (2018) 054.

[56] K. Zhou and B. Feng, Note on differential operators, CHY integrands, and unifying relations for amplitudes, J. High Energy Phys. 09 (2018) 160.

[57] M. Bollmann and L. Ferro, Transmuting CHY formulae, J. High Energy Phys. 01 (2019) 180.

[58] B. Feng, X. Li, and K. Zhou, Expansion of EYM theory by differential operators, Phys. Rev. D 100, 125012 (2019).

[59] A. Ntzi and M. Reiterer, Scattering amplitude annihilators, J. High Energy Phys. 02 (2020) 020.

[60] J. Broedel, O. Schlotterer, S. Stieberger, and Polylogarithms, Multiple zeta values and superstring amplitudes, Fortschr. Phys. 61, 812 (2013).

[61] S. Mizera and G. Zhang, A string deformation of the ParkeTaylor factor, Phys. Rev. D 96, 066016 (2017).

[62] S. He, F. Teng, and Y. Zhang, String Amplitudes from Fieldtheory Amplitudes and Vice Versa, Phys. Rev. Lett. 122, 211603 (2019).

[63] S. He, F. Teng, and Y. Zhang, String correlators: Recursive expansion, integration-by-parts and scattering equations, J. High Energy Phys. 09 (2019) 085.

[64] S. He, L. Ren, and Y. Zhang, Notes on polytopes, amplitudes and boundary configurations for Grassmannian string integrals, J. High Energy Phys. 04 (2020) 140.

[65] S. G. Naculich, Scattering equations and BCJ relations for gauge and gravitational amplitudes with massive scalar particles, J. High Energy Phys. 09 (2014) 029.

[66] S. G. Naculich, CHY representations for gauge theory and gravity amplitudes with up to three mass particles, J. High Energy Phys. 05 (2015) 050.

[67] S. G. Naculich, Amplitudes for massive vector and scalar bosons in spontaneously-broken gauge theory from the CHY representation, J. High Energy Phys. 09 (2015) 122.

[68] C. S. Lam and Y.-P. Yao, Off-shell CHY amplitudes, Nucl. Phys. B907, 678 (2016).

[69] L. Dolan and P. Goddard, Off-shell CHY amplitudes and Feynman graphs, J. High Energy Phys. 04 (2020) 141.

[70] C. S. Lam, Off-shell Yang-Mills amplitude in the CHY formalism, Phys. Rev. D 100, 045009 (2019).

[71] S. Weinzierl, Fermions and the scattering equations, J. High Energy Phys. 03 (2015) 141.

[72] S. He and Y. Zhang, Connected formulas for amplitudes in Standard Model, J. High Energy Phys. 03 (2017) 093.

[73] J. J. M. Carrasco, C. R. Mafra, and O. Schlotterer, Abelian Z-theory: NLSM amplitudes and Alpha'-corrections from the open string, J. High Energy Phys. 06 (2017) 093.

[74] O. Schlotterer and O. Schnetz, Closed strings as singlevalued open strings: A genus-zero derivation, J. Phys. A 52, 045401 (2019).

[75] N. E. J. Bjerrum-Bohr, P. H. Damgaard, and P. Vanhove, Minimal Basis for Gauge Theory Amplitudes, Phys. Rev. Lett. 103, 161602 (2009).

[76] S. Stieberger, Open \& closed vs. pure open string disk amplitudes, arXiv:0907.2211. 